\title{
Biorthogonal Partners and Applications
}

\author{
P. P. Vaidyanathan, Fellow, IEEE, and Bojan Vrcelj, Student Member, IEEE
}

\begin{abstract}
Two digital filters $H(z)$ and $F(z)$ are said to be biorthogonal partners of each other if their cascade $H(z) F(z)$ satisfies the Nyquist or zero-crossing property. Biorthogonal partners arise in many different contexts such as filterbank theory, exact and least squares digital interpolation, and multiresolution theory. They also play a central role in the theory of equalization, especially, fractionally spaced equalizers in digital communications. In this paper, we first develop several theoretical properties of biorthogonal partners. We also develop conditions for the existence of biorthogonal partners and FIR biorthogonal pairs and establish the connections to the Riesz basis property. We then explain how these results play a role in many of the above-mentioned applications.
\end{abstract}

\section{INTRODUCTION}

$\mathbf{T}$ WO DIGITAL filters $H(z)$ and $F(z)$ are said to be biorthogonal partners of each other if their cascade $H(z) F(z)$ satisfies the Nyquist or zero-crossing property. Biorthogonal partners ${ }^{1}$ arise in many different contexts such as filterbank theory [1], [23], [27], exact and least-squares digital interpolation [20], sampling theory [22], and multiresolution theory [10]. They also play a central role in the theory of equalization, especially fractionally spaced equalizers in digital communications [17]. In this paper, we first develop several theoretical properties of biorthogonal partners. We then explain the above-mentioned applications that use this concept directly or indirectly.

\section{A. Outline and Relation to Past Work}

The paper contains several new results and new proofs of well-known results. One main contribution here is to glue together certain widely known ideas in a unified manner under one cover. In Section II, we introduce the precise definition of biorthogonal partners. We derive a general closed-form expression for a filter $H(z)$ to be a biorthogonal partner of $F(z)$. We also develop a set of necessary and sufficient conditions on an FIR or IIR transfer function $F(z)$ such that there exists an FIR biorthogonal partner $H(z)$. This section also provides a deeper discussion on the existence of biorthogonal partners. In Section III, we study the application of these ideas in digital interpolation. This application also reveals the conditions on $F(z)$ that allow the existence of a biorthogonal partner. It also places in

Manuscript received April 21, 2000; revised January 29, 2001. This work was supported in part by the Office of Naval Research under Grant N00014-99-1-1002 and by Microsoft Research, Redmond, WA. The associate editor coordinating the review of this paper and approving it for publication was Prof. Hideaki Sakai.

The authors are with the Department of Electrical Engineering, California Institute of Technology, Pasadena, CA 91125 USA (e-mail: ppvnath@ sys.caltech.edu).

Publisher Item Identifier S 1053-587X(01)03347-5.

${ }^{1}$ The term "biorthogonal partner" has not been used in the past. We use it here because of the frequent need for a descriptive term. evidence the connection to linear independence and Riesz basis property of the shifted impulse responses $\{f(n-M k)\}$ of $F(z)$. This work is closely related to the concept of oblique projections studied extensively by Aldroubi et al. and Cohen et al. [2]-[5].

Section $\mathrm{V}$ reviews the role of biorthogonal partners in the least squares approximation of signals using interpolation models. Although this idea originated in the context of spline interpolation [7], its efficient implementation became possible because of the work by Unser et al. [20], [21], who developed fundamental digital filter structures for efficient implementation of the same. Applications of biorthogonal partners in the interpolation of signals based on continuous time models (e.g., spline models [13], [19]) is also discussed in Section VI. We also show that an all-FIR spline interpolation is sometimes possible, unlike the more well-known methods of Unser et al., which use an IIR/FIR combination [19]. The role of biorthogonal partners in multiresolution theory is described in Section VI-C. We show in particular that an FIR method for the computation of multiresolution coefficients is possible, without resorting to the traditional high degree of oversampling. Finally, in Section VII, we review applications in the theory of fractionally spaced equalizers for digital communications [12], [17].

\section{B. Notations}

Unless mentioned otherwise, all notations are as in [23]. We use the notations $[x(n)]_{\downarrow M}$ and $[X(z)]_{\downarrow M}$ to denote the decimated version $x(M n)$ and its $z$-transform. The expanded version

$$
\begin{cases}x(n / M), & n=\text { mul of } M \\ 0, & \text { otherwise }\end{cases}
$$

is similary denoted by $[x(n)]_{\uparrow M}$, and its $z$-transform $X\left(z^{M}\right)$ is denoted by $[X(z)]_{\uparrow M}$. Notice that

$$
\begin{aligned}
{\left[X\left(z^{M}\right) Y(z)\right]_{\downarrow M} } & =X(z)[Y(z)]_{\downarrow M} \quad \text { so that } \\
{\left[X\left(z^{M}\right) Y(z)\right]_{\downarrow M \uparrow M} } & =X\left(z^{M}\right)[Y(z)]_{\downarrow M \uparrow M} .
\end{aligned}
$$

The tilde notation $\tilde{F}(z)$ is defined by $\tilde{F}(z)=F^{*}\left(1 / z^{*}\right)$ so that on the unit circle, $\tilde{F}(z)=F^{*}\left(e^{j \omega}\right)$. Thus, $\tilde{F}(z) F(z)$ evaluated on the unit circle is the magnitude square function. In situations where the $z$-transform does not exist in the conventional sense (e.g., ideal filters), the notation $z$ stands for $e^{j \omega}$ so that $H(z)$ is the frequency response $H\left(e^{j \omega}\right)$.

\section{Biorthogonal PARTNERS: DEFINITION AND PROPERTIES}

Two transfer functions $F(z)$ and $H(z)$ are said to form a biorthogonal pair with respect to an integer $M$ if

$$
[H(z) F(z)]_{\downarrow M}=1 .
$$




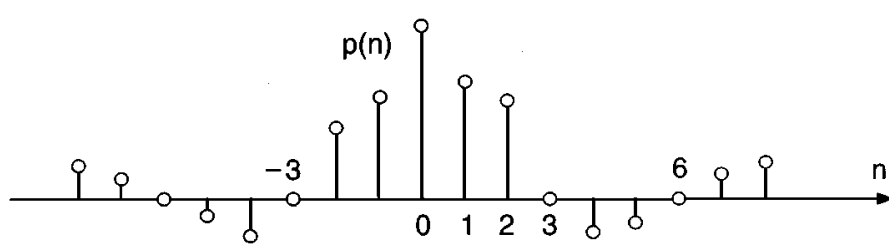

Fig. 1. Nyquist $(M)$ property of $P(z)$ demonstrated for $M=3$.

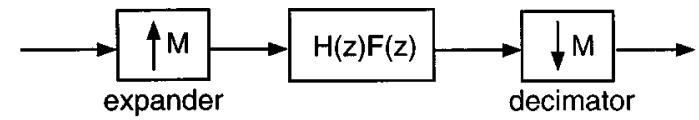

(a)

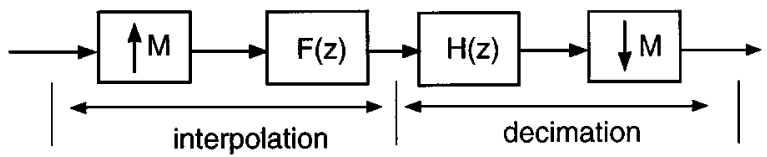

(b)

Fig. 2. Interpretation of biorthogonal partners $H(z), F(z)$ in terms of signal flowgraphs.

We say that $H(z)$ is a biorthogonal partner (or just partner) of $F(z)$. Notice that if $M$ is changed, the two filters may not remain partners. The term "with respect to $M$ " is usually understood from the context and is never mentioned unless there is a possible confusion. Evidently, $H(z)$ and $F(z)$ can be interchanged without altering this property. Equation (1) is equivalent to the statement that the impulse response $p(n)$ of the product filter $P(z)=H(z) F(z)$ satisfies the $\operatorname{Nyquist}(M)$ condition

$$
p(M n)=\delta(n)
$$

That is, the $M$-fold decimation of $p(n)$ yields the impulse $\delta(n)$ (Fig. 1). We can regard $H(z)$ and $F(z)$ as any pair that defines a factorization of a Nyquist $(M)$ filter $P(z)$.

Notice that every pair of filters $\left\{H_{k}(z), F_{k}(z)\right\}$ in an $M$-channel perfect reconstruction (PR or biorthogonal) filterbank satisfies this condition. This is also the reason for the phrase "biorthogonal pair." Recall that the "multirate" system shown in Fig. 2(a) is just an LTI (single rate) system with transfer function $[H(z) F(z)]_{\downarrow M}=1$ (see "polyphase identity" [23]). Thus, the implication of (1) is that the system shown in Fig. 2(a) is an identity system. That is, the decimation filter $[H(z)$ followed by $\downarrow M]$ is diagrammatically the right inverse of the interpolation filter [ $\uparrow M$ followed by $F(z)$ ] [Fig. 2(b)].

Given a transfer function $F(z)$ and the integer $M$, does a biorthogonal partner $H(z)$ always exist? When is it unique? If $F(z)$ is FIR, then under what conditions does there exist an FIR biorthogonal partner $H(z)$ ? For rational $F(z)$, can we get FIR partners? In this section, we answer these questions.

\section{A. General Expression}

We first derive a general expression for $H(z)$ in terms of $F(z)$. In the following theorem, note that the notation $[G(z) F(z)]_{\downarrow M \uparrow M}$ stands for $Q\left(z^{M}\right)$, where $Q(z) \triangleq[G(z) F(z)]_{\downarrow M}$.

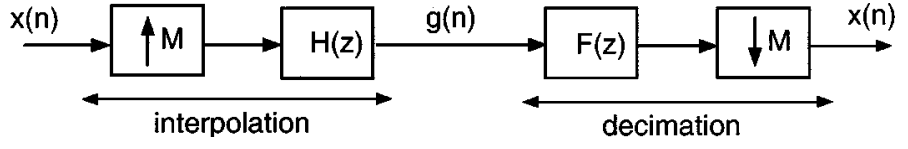

(a)

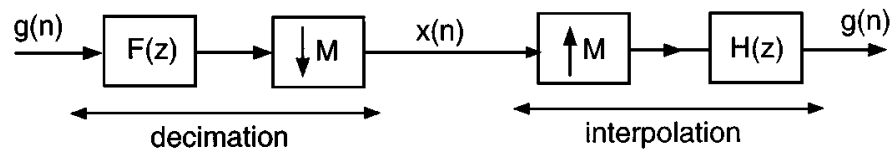

(b)

Fig. 3. Pertaining to the proof of Theorem 1.

Theorem 1-General Form of Biorthogonal Partner: The transfer function $H(z)$ satisfies $[H(z) F(z)]_{\downarrow M}=1$ if and only if it can be expressed in the form

$$
H(z)=\frac{G(z)}{[G(z) F(z)]_{\downarrow M \uparrow M}}
$$

for some transfer function $G(z)$. we have

Proof: Given a transfer function $H(z)$ of the above form,

$$
\begin{aligned}
{[H(z) F(z)]_{\downarrow M} } & =\left[\frac{G(z) F(z)}{[G(z) F(z)]_{\downarrow M \uparrow M}}\right]_{\downarrow M} \\
& =\frac{[G(z) F(z)]_{\downarrow M}}{[G(z) F(z)]_{\downarrow M}}=1
\end{aligned}
$$

which proves the "if part." Conversely, suppose $H(z)$ is such that $[H(z) F(z)]_{\downarrow M}=1$. Consider the interpolation scheme shown in Fig. 3(a), where $x(n)$ is an arbitrary input and $g(n)$ the output of $H(z)$. If we cascade $F(z)$ and the decimator $\downarrow M$ as shown in the figure, then the output is $x(n)$ because $[H(z) F(z)]_{\downarrow M}=1$. The important point is that this also means that the signal $g(n)$ input to the system of Fig. 3(b) comes out as $g(n)$. This is because $g(n)$ by definition is the output of the left half in Fig. 3(a) driven by $x(n)$. Thus

$$
[G(z) F(z)]_{\downarrow M \uparrow M} H(z)=G(z)
$$

which indeed can be rewritten as (2).

$\nabla \nabla \nabla$

Notice in the proof that since $X(z)=[G(z) F(z)]_{\downarrow M}$ is arbitrary, it can be chosen so that the denominator of (2) is nonzero for all $z=e^{j \omega}$. In general, a biorthogonal partner may or may not exist, and when it exists, it may not be unique. It follows from Theorem 1 that a stable biorthogonal partner can be found if and only if there exists a $G(z)$ such that $\left[G\left(e^{j \omega}\right) F\left(e^{j \omega}\right)\right]_{\downarrow M}$ is nonzero for all $\omega$. We will return to a more insightful discussion of existence issues in Section IV. Here are some special situations of interest.

1) If $\left|F\left(e^{j \omega}\right)\right|^{2}>0$ for all $\omega$, then $H(z)=1 / F(z)$ is theoretically stable (though not necessarily causal). This is conceptually the simplest biorthogonal partner.

2) If $F(z)$ has unit circle zeros, then $H(z)=1 / F(z)$ is not a stable filter. However, we can often get other solutions. For example, suppose the decimated version [23]

$$
\left[F\left(e^{j \omega}\right)\right]_{\downarrow M}=\frac{1}{M} \sum_{k=0}^{M-1} F\left(e^{j(\omega-2 \pi k) / M}\right)
$$




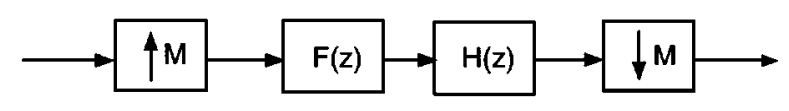

(a)

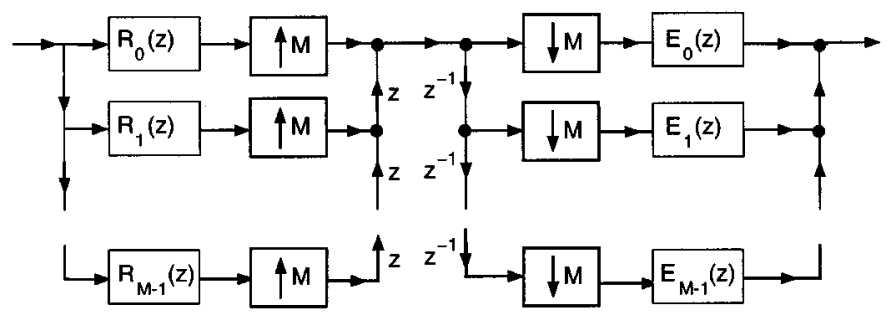

(b)

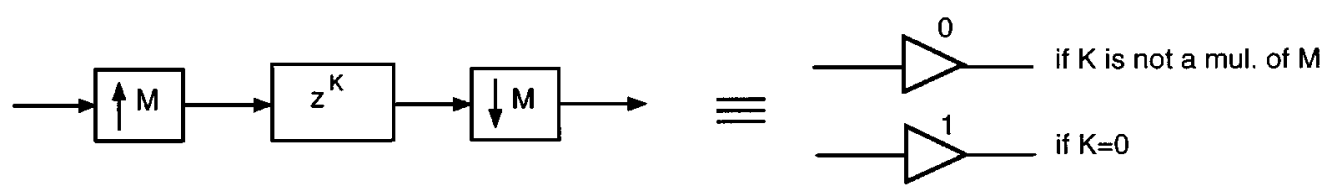

(c)

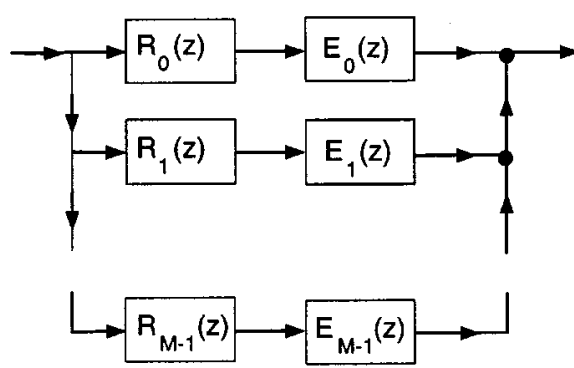

(d)

Fig. 4. Block diagram interpretation of the proof of Theorem 2. (a) Cascade of interpolator and decimator. (b) Redrawing in polyphase form. (c) Basic multirate identity. (d) Simplification of part (b) using the identity.

is nonzero for all $\omega$. Then, we can set $G(z)=1$ in (2) to obtain the stable biorthogonal partner

$$
H(z)=\frac{1}{[F(z)]_{\downarrow M \uparrow M}} .
$$

3) The preceding examples show that the biorthogonal partner $H(z)$ in general is not unique. To get yet another solution, consider the filter

$$
H(z)=\frac{\tilde{F}(z)}{[\tilde{F}(z) F(z)]_{\downarrow M \uparrow M}} .
$$

Since $\tilde{F}(z)=F^{*}\left(1 / z^{*}\right)$, we have $\tilde{F}\left(e^{j \omega}\right)=F^{*}\left(e^{j \omega}\right)$ so that $\tilde{F}(z) F(z)=\left|F\left(e^{j \omega}\right)\right|^{2}$ on the unit circle. The preceding solution is a special case of (2) with $G(z)=$ $\tilde{F}(z)$ and works as long as $\left[\tilde{F}\left(e^{j \omega}\right) F\left(e^{j \omega}\right)\right]_{\downarrow M}$ is nonzero for all $\omega$. This is a "popular solution" in some sense and is described in greater detail in Section $\mathrm{V}$ in the context of least squares approximations.

4) If $H_{1}(z)$ and $H_{2}(z)$ are biorthogonal partners of $F(z)$, then so is the convex combination $\alpha H_{1}(z)+(1-$ a) $H_{2}(z)$.

5) Suppose $F\left(e^{j \omega}\right)$ is nonzero only in a set of measure < $2 \pi / M$ in $0 \leq \omega<2 \pi$ [e.g., $F(z)$ ideal lowpass with total passband width $<2 \pi / M]$. Then, $H\left(e^{j \omega}\right) F\left(e^{j \omega}\right)$ has the same property so that

$$
\left[H\left(e^{j \omega}\right) F\left(e^{j \omega}\right)\right]_{\downarrow M}
$$

cannot fill the region $0 \leq \omega<2 \pi$ completely. There does not exist a biorthogonal partner for this $F\left(e^{j \omega}\right)$.

6) If we replace $G(z)$ with $G(z) S\left(z^{M}\right)$ in (2), then the $S(z)$ part merely cancels and leaves $H(z)$ unchanged. Therefore, $G(z)$ is not unique for a given $H(z), F(z)$ pair. If $G_{1}(z)$ and $G_{2}(z)$ are two possible choices, then we can verify that $G_{1}(z)=C\left(z^{M}\right) G_{2}(z)$ for some $C(z)$ [to see this, just divide one representation of $H(z)$ by the other].

\section{B. Rational and FIR Cases}

In practice the situation of most interest is the case where $F(z)$ is rational, that is, $F(z)=A(z) / B(z)$ where $A(z)$ and $B(z)$ are polynomials in $z^{-1}$. In this case the trivial choice $H(z)=B(z) / A(z)$ gives a rational biorthogonal partner. This is stable (though possibly noncausal) as long as $A(z)$ has no unit circle zeros. If $F(z)$ is FIR, then the trivial choice $H(z)=$ $1 / F(z)$ yields an allpole IIR filter. A question of significant interest is this: if $F(z)$ is FIR, can we obtain an FIR solution $H(z)$ ? The answer is provided below. This is key to some of the applications described in the next several sections. In fact, this theorem is applicable in nearly the same form in the theory of fractionally spaced equalizers [15]-[17].

Theorem 2-Existence of FIR Partner: Suppose $F(z)$ is FIR. Express it in the polyphase form $F(z)=$ $\sum_{k=0}^{M-1} z^{k} R_{k}\left(z^{M}\right)$. Then, there exists an FIR filter $H(z)$ such that $[H(z) F(z)]_{\downarrow M}=1$ if and only if the greatest common divisor (gcd) $C(z)$ of the $M$ polyphase components 
$\left\{R_{k}(z)\right\}$ is trivial, i.e., has the form $C(z)=c z^{-N}$ for some constant $c$ and integer $N$.

Example 1: Thus, the gcd should be no more sophisticated than a delay. Given an arbitrary FIR transfer function $F(z)$, this gcd condition is nearly always satisfied. For example, let

$$
F(z)=z^{-1}+4 z^{-2}+z^{-3}
$$

and $M=2$. We can write $F(z)=4 z^{-2}+z\left(z^{-2}+z^{-4}\right)$, which shows that $R_{0}(z)=4 z^{-1}$ and $R_{1}(z)=z^{-1}+z^{-2}$. These two polynomials have no common factors other than delays and constant so that the conditions of the theorem are satisfied. Indeed, we can readily verify that the FIR filter $H(z)=z^{3}-0.25 z^{4}$ is an FIR biorthogonal partner of $F(z)$.

Proof of Theorem 2: If the polyphase components $\left\{R_{k}(z)\right\}$ have gcd equal to unity, then there exist FIR filters $\left\{E_{k}(z)\right\}$ such that $\sum_{k=0}^{M-1} E_{k}(z) R_{k}(z)=1$. These can be constructed using a generalization of Euclid's algorithm [23]. If the gcd is $c z^{-N}$, then the same is true because the constant $c$ and $z^{-N}$ can be absorbed into $E_{k}(z)$ anyway. Now, define $H(z)$ to be the FIR filter

$$
H(z)=\sum_{k=0}^{M-1} z^{-k} E_{k}\left(z^{M}\right) .
$$

Then, $H(z) F(z)$ has the form $\sum_{i=0}^{M-1} z^{i} S_{i}\left(z^{M}\right)$, where $S_{0}(z)=\sum_{k=0}^{M-1} E_{k}(z) R_{k}(z)$. Thus,

$$
[H(z) F(z)]_{\downarrow M}=S_{0}(z)=\sum_{k=0}^{M-1} E_{k}(z) R_{k}(z)=1
$$

which shows that $H(z)$ is an FIR biorthogonal partner. Conversely, suppose there exists an FIR biorthogonal partner $H(z)$. Defining its polyphase components as $E_{k}(z)$ [i.e., $H(z)=\sum_{k=0}^{M-1} z^{-k} E_{k}\left(z^{M}\right)$ ], we have $[H(z) F(z)]_{\downarrow M}=\sum_{k=0}^{M-1} E_{k}(z) R_{k}(z)=1$. If the $\mathrm{gcd}$ of $\left\{R_{k}(z)\right\}$ is $C(z)$, then this can be written as

$$
[H(z) F(z)]_{\downarrow M}=C(z) \sum_{k=0}^{M-1} E_{k}(z) \hat{R}_{k}(z)=1
$$

where $E_{k}(z)$ and $\hat{R}_{k}(z)$ are FIR. The preceding equation says that the product of two FIR filters $C(z)$ and $\sum_{k=0}^{M-1} E_{k}(z) \hat{R}_{k}(z)$ is unity. This is not possible unless $C(z)$ has the form $c z^{-N}$.

$\nabla \nabla \nabla$

A block diagram interpretation of this proof is insightful. Recall that $[H(z) F(z)]_{\downarrow M}=1$ is equivalent to the statement that Fig. 4(a) is an identity system. The polyphase representation of this is shown in Fig. 4(b). We now use the identity shown in Fig. 4(c) (see polyphase identity [23]) to simplify Fig. 4(b) to Fig. 4(d). Thus, $[H(z) F(z)]_{\downarrow M}=1$ is completely equivalent to the statement that the parallel connection shown in Fig. 4(d) must be identity. If $R_{k}(z)$ are FIR with no overall common factor, then we can indeed find FIR filters $E_{k}(z)$ so that this is an identity system.

Corollary 1-FIR Partner for IIR Filter: Suppose $F(z)=$ $A(z) / B(z)$, which is the most general rational IIR form. As-

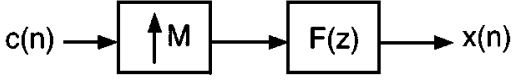

(a)

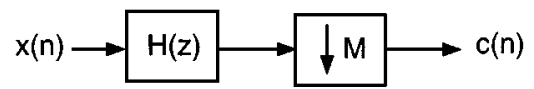

(b)

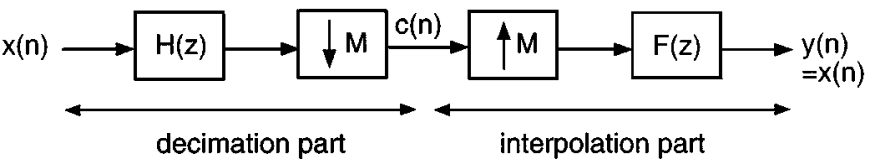

(c)

Fig. 5. (a) Signal model. (b) Computation of the coefficients $c(n)$ in the model for $x(n)$. (c) Complete system looking like a subband channel in an $M$-band filterbank.

sume that the numerator $A(z)$ has an FIR biorthogonal partner $H_{a}(z)$ so that

$$
\left[H_{a}(z) A(z)\right]_{\downarrow M}=1 .
$$

Then, $H(z)=H_{a}(z) B(z)$ is an FIR partner for the IIR filter $F(z)$. This is because $H(z) F(z)=H_{a}(z) A(z)$ so that $[H(z) F(z)]_{\downarrow M}=\left[H_{a}(z) A(z)\right]_{\downarrow M}=1$ from (3).

\section{Discrete-Time Signal Models}

Consider a discrete-time signal $x(n)$ that can be modeled as the output of a digital interpolation filter, as shown in Fig. 5(a). In this model, $F(z)=\sum_{n} f(n) z^{-n}$ is a fixed digital filter. We assume $f(n)$ and $c(n)$ are $\ell_{2}$ sequences (finite-energy sequences). By appropriately choosing $c(n)$, we can generate a whole class of signals $x(n)$ like this. This class $\mathcal{F}$ forms a subspace of $\ell_{2}$. Since

$$
x(n)=\sum_{k} c(k) f(n-k M)
$$

this is the subspace spanned by the set of sequences $\left\{\eta_{k}(n)\right\} \triangleq\{f(n-k M)\}$. Note that with real-time dimensions, the samples of $x(n)$ are spaced apart more closely ${ }^{2}$ than those of $c(n)$.

Given a signal $x(n)$ in the subspace $\mathcal{F}$, how do we compute the coefficients $c(n)$ [i.e., the correct driving signal in Fig. 5(a)]? Assuming that a biorthogonal partner exists for $F(z)$, all we have to do is to filter $x(n)$ through $H(z)$ and decimate the output, as shown in Fig. 5(b). To see this, simply note that the output of the decimator is

$$
\begin{aligned}
{[X(z) H(z)]_{\downarrow M} } & =\left[C\left(z^{M}\right) F(z) H(z)\right]_{\downarrow M} \\
& =C(z) \times[F(z) H(z)]_{\downarrow M}=C(z)
\end{aligned}
$$

because $[F(z) H(z)]_{\downarrow M}=1$. As seen in Section II-A, the biorthogonal partner $H(z)$ is not unique, but any such $H(z)$ will do. Fig. 5(c) shows the decimation system for generating $c(n)$ and the interpolation system for generating $x(n)$ cascaded together. It is clear that $c(n)$ can be regarded as one subband signal of an $M$-band biorthogonal filterbank with input $x(n)$.

\footnotetext{
${ }^{2}$ In general, $c(n)$ is not a subsampled or decimated version of $x(n)$. However, the model allows us to recover $x(n)$ from its $M$-fold decimated version $x(M n)$ under mild conditions [25], [26].
} 


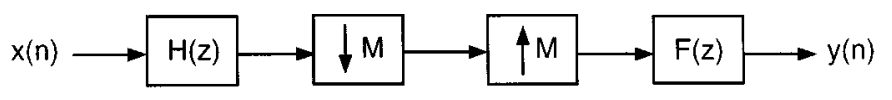

Fig. 6. Pertaining to Lemma 1.

If $F(z)$ is FIR with an FIR biorthogonal partner $H(z)$, all computations are FIR based.

Lemma 1: Consider the interconnection of Fig. 6, and assume $F(z)$ is rational (FIR or IIR). If $H(z)$ is such that for any input $x(n) \in \mathcal{F}$ [i.e., any $x(n)$ with $X(z)=C\left(z^{M}\right) F(z)$ ], the final output $y(n)$ is exactly equal to $x(n)$, then $H(z)$ is necessarily a biorthogonal partner of $F(z)$. The assertion is not true when nonrational $F(z)$ (e.g., ideal brickwall filter) is allowed. $\diamond$

Proof: The filter $H(z)$ in the Lemma is such that if an input of the form $X(z)=C\left(z^{M}\right) F(z)$ is applied at the left in Fig. 6, then the output of $F(z)$ is also $X(z)$. That is, the following equation holds:

$$
\left(\left[C\left(z^{M}\right) F(z) H(z)\right]_{\downarrow M \uparrow M}\right) \times F(z)=C\left(z^{M}\right) F(z) .
$$

Using standard multirate identities, we can factor out $C\left(z^{M}\right)$ from the left side and write this as

$$
C\left(z^{M}\right)\left([F(z) H(z)]_{\downarrow M \uparrow M}\right) \times F(z)=C\left(z^{M}\right) F(z) .
$$

If this holds for all $C(z)$ such that $c(n) \in \ell_{2}$, we can cancel $C\left(z^{M}\right)$ from both sides. Since $F(z)$ is rational, it can be cancelled as well, proving that $[F(z) H(z)]_{\downarrow M}=1$, that is, $H(z)$ is a biorthogonal partner of $F(z)$.

If $F(z)$ is allowed to be nonrational, the cancellation step is not valid. In fact, we can produce a counterexample showing that the same assertion is not true. Let $F\left(e^{j \omega}\right)$ be lowpass as in Fig. 7(a). For any $H\left(e^{j \omega}\right)$, the support of $H\left(e^{j \omega}\right) F\left(e^{j \omega}\right)$ is restricted to $|\omega|<\pi / 4$ so that $\left[H\left(e^{j \omega}\right) F\left(e^{j \omega}\right)\right]_{\downarrow 2}$ is zero for $\pi / 2<\omega<3 \pi / 2$, as demonstrated in Fig. 7(b). The filter $F(z)$ therefore has no biorthogonal partner for $M=2$. However, the choice $H\left(e^{j \omega}\right)=2 F\left(e^{j \omega}\right)$ is such that if $x(n) \in$ $\mathcal{F}$ is applied to $H(z)$ in Fig. 6, the output of $F(z)$ is indeed $x(n)$. This is because, even though $[F(z) H(z)]_{\downarrow 2} \neq 1$, we have $F(z)[F(z) H(z)]_{\downarrow 2 \uparrow 2}=F(z)$ in this example, so that

$$
\begin{aligned}
Y(z) & =\left(\left[C\left(z^{2}\right) F(z) H(z)\right]_{\downarrow 2 \uparrow 2}\right) \times F(z) \\
& =C\left(z^{2}\right)\left([F(z) H(z)]_{\downarrow 2 \uparrow 2}\right) \times F(z) \\
& =C\left(z^{2}\right) F(z)=X(z)
\end{aligned}
$$

indeed.

\section{EXISTENCE OF BIORTHOgONAL PARTNERS}

From Theorem 1, we know that $F(z)$ has a stable biorthogonal partner if there exists $G(z)$ such that $[G(z) F(z)]_{\downarrow M}$ has no unit circle zeros. It is insightful to look at the existence issue in different ways, as we will do in this section.

Lemma 2: Suppose $F(z)$ has a biorthogonal partner $H(z)$ so that $[H(z) F(z)]_{\downarrow M}=1$. Then, the signals

$$
\eta_{k}(n) \triangleq f(n-k M)
$$

are linearly independent.

Proof: If $\eta_{k}(n)$ are linearly dependent, then there exists a sequence $c(n)$ that is not zero for all $n$, such that $\sum_{k} c(k) f(n-$

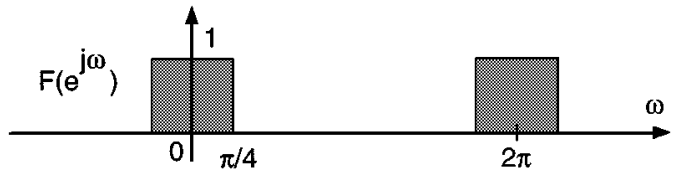

(a)

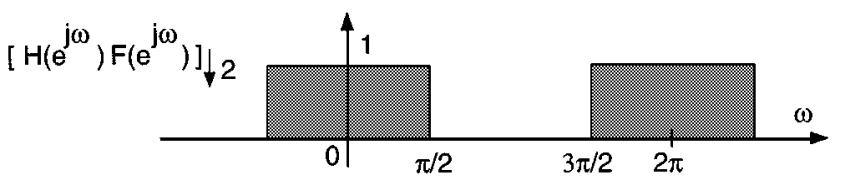

(b)

Fig. 7. (a) Filter $F\left(e^{j \omega}\right)$ in the model and (b) $\left[H\left(e^{j \omega}\right) F\left(e^{j \omega}\right)\right]_{\downarrow 2}$ for $H\left(e^{j \omega}\right)=2 F\left(e^{j \omega}\right)$.

$k M)=0$. In $z$-transform notation, this means $C\left(z^{M}\right) F(z)=$ 0 . Thus

$0=\left(C\left(z^{M}\right) F(z) H(z)\right)_{\downarrow M}=C(z) \times(F(z) H(z))_{\downarrow M}=C(z)$

because $[F(z) H(z)]_{\downarrow M}=1$. This shows that $C(z)=0$, contradicting the assumption that $c(n)$ is not the zero sequence. $\nabla \nabla \nabla$

Corollary 2: In Fig. 6, suppose that $H(z)$ is such that any input $x(n) \in \mathcal{F}$ produces the output $y(n)=x(n)$. In general, this does not mean that $H(z)$ is a biorthogonal partner because such a partner may not even exist (Lemma 1). However, if $F(z)$ does have a biorthogonal partner, then $H(z)$ is such a partner. $\diamond$

Proof: Choose $X(z)=F(z) \in \mathcal{F}$. Then, $Y(z)=F(z)$ as well so that

$$
F(z)=\left([F(z) H(z)]_{\downarrow M \uparrow M}\right) F(z) .
$$

Thus, $\left(D\left(z^{M}\right)-1\right) F(z)=0$, where $D(z) \triangleq[F(z) H(z)]_{\downarrow M}$. If there exists a biorthogonal partner for $F(z)$, then by the linear independence asseted by Lemma 2 , it follows that $D\left(z^{M}\right)-1=$ 0 , that is, $D(z)=[F(z) H(z)]_{\downarrow M}=1$. In other words, $H(z)$ is a biorthogonal partner of $F(z)$.

$\nabla \nabla \nabla$

Lemma 3: Suppose $F(z)$ is stable [i.e., $\sum_{n}|f(n)|<\infty$ ] and is such that the set of sequences $\left\{\eta_{k}(n)\right\}$ defined in (4) is linearly independent in the sense that $\sum_{k} c(k) f(n-k M)$ cannot be arbitrarily small for any $c(n)$ with fixed nonzero energy. More precisely, suppose there exists $A>0$ such that ${ }^{3}$

$$
\sum_{n}\left|\sum_{k} c(k) f(n-k M)\right|^{2} \geq A \sum_{n}|c(n)|^{2} .
$$

Then, $F(z)$ has a biorthogonal partner $H(z)$.

Proof: We will show that (5) implies that $\left(\left|F\left(e^{j \omega}\right)\right|^{2}\right)_{\downarrow M}$ $>0$ for all $\omega$. Then, the filter

$$
H\left(e^{j \omega}\right) \triangleq \frac{F^{*}\left(e^{j \omega}\right)}{\left(\left|F\left(e^{j \omega}\right)\right|^{2}\right)_{\downarrow M \uparrow M}}
$$

is a biorthogonal partner [set $G\left(e^{j \omega}\right)=F^{*}\left(e^{j \omega}\right)$ in Theorem 1]. To show that $\left(\left|F\left(e^{j \omega}\right)\right|^{2}\right)_{\downarrow M}>0$, assume the contrary, that is, $\left(\left|F\left(e^{j \omega_{1}}\right)\right|^{2}\right)_{\downarrow M}=0$ for some $\omega_{1}$. That is

$$
\sum_{k=0}^{M-1}\left|F\left(e^{j\left(\left(\omega_{1}+2 \pi k\right) / M\right)}\right)\right|^{2}=0 .
$$

${ }^{3}$ Note that this is one of the two conditions for $\{f(n-k M)\}$ to be a Riesz basis [6], [24]. 


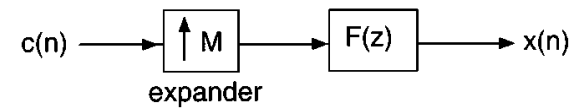

(a)

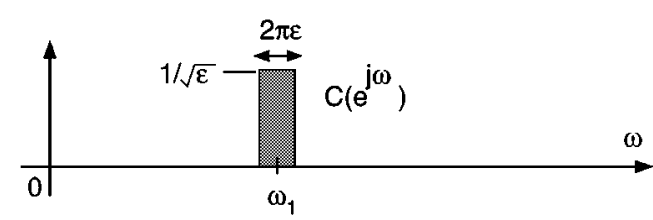

(b)

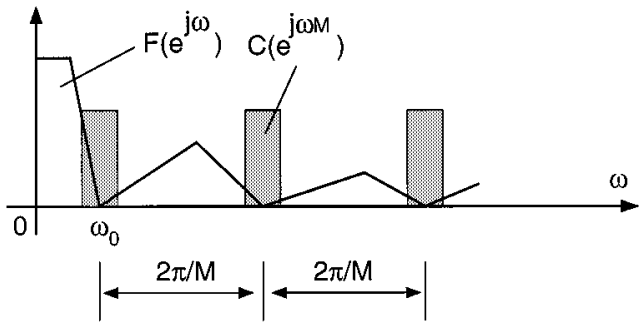

(c)

Fig. 8. Pertaining to the proof of Lemma 3. (a) Interpolation filter. (b) Narrowband unit-energy input. (c) Filter $F\left(e^{j \omega}\right)$ and its input $C\left(e^{j \omega M}\right)$.

Since each term in the above summation is non-negative, this implies $F\left(e^{j\left(\left(\omega_{1}+2 \pi k\right) / M\right)}\right)=0$ for each $k$. That is

$$
F\left(e^{j\left(\omega_{0}+2 \pi k / M\right)}\right)=0, \quad 0 \leq k \leq M-1
$$

where $\omega_{0}=\omega_{1} / M$. Now, consider the interpolation scheme in Fig. 8(a). Choose $c(n)$ such that $C\left(e^{j \omega}\right)$ is the pulse shown in Fig. 8(b), with energy concentrated around $\omega_{1}$. Note that the energy $\sum_{n}|c(n)|^{2}=\int_{0}^{2 \pi}\left|C\left(e^{j \omega}\right)\right|^{2} d \omega / 2 \pi=1$ for any $\epsilon$. The output of the expander, which is $C\left(e^{j \omega M}\right)$, evidently has the same energy, but it is distributed around the frequencies $\left(\omega_{0}+\right.$ $2 \pi k / M)$. If we make $\epsilon$ arbitrarily small, the energy of $x(n)$, namely

$$
\sum_{n}\left|\sum_{k} c(k) f(n-k M)\right|^{2}
$$

is concentrated increasingly more around the zeros of $F\left(e^{j \omega}\right)$. Since $\sum_{n}|f(n)|<\infty$, the response $F\left(e^{j \omega}\right)$ is a continuous function, and so is $\left|F\left(e^{j \omega}\right)\right|$. Therefore, the energy of $x(n)$ can be made arbitrarily small, although the energy of $c(n)$ remains unity. This means there cannot exist $A>0$ satisfying (5). Summarizing, if (5) is satisfied, then $\left(\left|F\left(e^{j \omega}\right)\right|^{2}\right)_{\downarrow M}>0$ for all $\omega$.

$\nabla \nabla \nabla$

Connection to Riesz Basis: The condition that $F(z)$ be stable in Lemma 3 implies, in particular, that $\left|F\left(e^{j \omega}\right)\right|^{2}<B$ for some $B<\infty$. Therefore, $\int\left|X\left(e^{j \omega}\right)\right|^{2} d \omega / 2 \pi=$ $\int\left|F\left(e^{j \omega}\right) C\left(e^{j \omega M}\right)\right|^{2} d \omega / 2 \pi \leq B \int\left|C\left(e^{j \omega}\right)\right|^{2} d \omega / 2 \pi$. Since $x(n)=\sum_{k} c(k) f(n-k M)$, this implies, by Parseval's relation, that

$$
\sum_{n}\left|\sum_{k} c(k) f(n-k M)\right|^{2} \leq B \sum_{n}|c(n)|^{2} .
$$

Thus, under the conditions of Lemma 3, both the preceding inequality and (5) are true, that is

$$
A \sum_{n}|c(n)|^{2} \leq \sum_{n}\left|\sum_{k} c(k) f(n-k M)\right|^{2} \leq B \sum_{n}|c(n)|^{2}
$$

where $A>0$ and $B<\infty$. This is precisely the definition of a Riesz basis. That is, under the conditions of Lemma 3, $\{f(n-k M)\}$ is a Riesz basis for the subspace $\mathcal{F}$ defined at the beginning of Section III.

The main points of the preceding two lemmas can be usefully summarized as follows. A digital filter $F(z)$ has a biorthogonal partner (with respect to integer $M$ ) if and only if $\eta_{k}(n) \triangleq f(n-$ $k M$ ) are linearly independent. From the technique of the proof of Lemma 3, we readily obtain the following.

Theorem 3-Existence of Biorthogonal Partners: The filter $F(z)$ has a biorthogonal partner if and only if $\left(\left|F\left(e^{j \omega}\right)\right|^{2}\right)_{\downarrow M}>$ 0 for all $\omega$. Thus, if there is a biorthogonal partner, then, in particular, the choice $H\left(e^{j \omega}\right)=F^{*}\left(e^{j \omega}\right) /\left[\left(\left|F\left(e^{j \omega}\right)\right|^{2}\right)_{\downarrow M \uparrow M}\right]$ will work. Moreover, define the new filters

$$
\begin{aligned}
& H_{1}\left(e^{j \omega}\right)=\frac{F^{*}\left(e^{j \omega}\right)}{\left(\left(\left|F\left(e^{j \omega}\right)\right|^{2}\right)_{\downarrow M \uparrow M}\right)^{1 / 2}} \\
& F_{1}\left(e^{j \omega}\right)=\frac{F\left(e^{j \omega}\right)}{\left(\left(\left|F\left(e^{j \omega}\right)\right|^{2}\right)_{\downarrow M \uparrow M}\right)^{1 / 2}} .
\end{aligned}
$$

Then, $H_{1}\left(e^{j \omega}\right)$ is a biorthogonal partner of $F_{1}\left(e^{j \omega}\right)$, and moreover, the set of signals $\left\{\eta_{k}(n)\right\} \triangleq\left\{f_{1}(n-k M)\right\}$ forms an orthonormal basis for the space $\mathcal{F}$ spanned by $\{f(n-k M)\} . \diamond$

Proof: If $\left(\left|F\left(e^{j \omega}\right)\right|^{2}\right)_{\downarrow M}>0$, then we can define $H\left(e^{j \omega}\right)=F^{*}\left(e^{j \omega}\right) /\left(\left|F\left(e^{j \omega}\right)\right|^{2}\right) \downarrow M \uparrow M$, and this is a biorthogonal partner. Conversely, if $F(z)$ has a biorthogonal partner, then the signals $\eta_{k}(n)=f(n-k M)$ are linearly independent (Lemma 2). This means $\left(\left|F\left(e^{j \omega}\right)\right|^{2}\right)_{\downarrow M}$ cannot be zero for any $\omega$ (otherwise, we can create an annihilating input as in the proof of Lemma 3 violating linear independence). The biorthogonality of $H_{1}(z)$ and $F_{1}(z)$ follows readily. Moreover, $\left|F_{1}\left(e^{j \omega}\right)\right|_{\downarrow M}^{2}=1$, that is, $\left|F_{1}\left(e^{j \omega}\right)\right|^{2}$ is $\operatorname{Nyquist}(M)$. In the time domain, this means $\sum_{n} f_{1}(n) f_{1}^{*}(n-k M)=\delta(k)$, which is equivalent to the orthonormality of $\left\{f_{1}(n-k M)\right\} \cdot \nabla \nabla \nabla$

For rational filters, we can replace $\left(\left(\left|F\left(e^{j \omega}\right)\right|^{2}\right)_{\downarrow M}\right)^{1 / 2}$ in the denominator of $F_{1}\left(e^{j \omega}\right)$ with a spectral factor of $(\tilde{F}(z) F(z))_{\downarrow M}$ to obtain an orthonormal basis. We then set $H_{1}(z)=\tilde{F}_{1}(z)$ as in any orthonormal filterbank. Notice that the orthogonalized filter $F_{1}(z)$ can be written as $F_{1}(z)=F(z) / D\left(z^{M}\right)$, where $D(z)$ is a spectral factor of $(\tilde{F}(z) F(z))_{\downarrow M}$. Thus

$X(z)=C\left(z^{M}\right) F(z)=C\left(z^{M}\right) D\left(z^{M}\right) F_{1}(z)=C_{1}\left(z^{M}\right) F_{1}(z)$.

That is, $x(n)=\sum_{k} c_{1}(k) f_{1}(n-k M)$; therefore, the coefficients of expansion in the new basis are $c_{1}(n)$.

Corollary 3-FIR Case: For FIR $F(z)$, there exists a biorthogonal partner if and only if $F(z)$ is free from factors of the form $\left(1-z^{-M} e^{j \omega_{1}}\right)$. These factors represent a set of $M$ zeros spaced uniformly on the unit circle. 


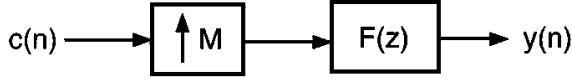

Fig. 9. Interpolation-filter model defining a class of signals $y(n)$.

Proof: If $F(z)$ has the factor $\left(1-z^{-M} e^{j \omega_{1}}\right)$, then $\left(\left|F\left(e^{j \omega}\right)\right|^{2}\right)_{\downarrow M}=\left|1-e^{-j\left(\omega-\omega_{1}\right)}\right|^{2} \times\left|F_{1}\left(e^{j \omega}\right)\right|^{2}$ for some FIR $F_{1}(z)$. This vanishes at $\omega=\omega_{1}$, violating $\left(\left|F\left(e^{j \omega}\right)\right|^{2}\right)_{\downarrow M}>0$. Conversely, if $\left(\left|F\left(e^{j \omega}\right)\right|^{2}\right)_{\downarrow M}>0$ is violated, there exists $\omega_{0}$ such that $F\left(e^{j\left(\omega_{0}+2 \pi k / M\right)}\right)=0$ for all $k$ (see proof of Lemma $3)$. That is, $F(z)$ has the factor

$$
\prod_{k=0}^{M-1}\left(1-z^{-1} e^{j \omega_{0}} e^{j 2 \pi k / M}\right)
$$

which can be rewritten as $\left(1-z^{-M} e^{j \omega_{1}}\right)$ for $\omega_{1}=M \omega_{0}$. Thus, the condition $\left(\left|F\left(e^{j \omega}\right)\right|^{2}\right) \downarrow M>0$ in Theorem 3 is equivalent to the nonexistence of factors of the form $\left(1-z^{-M} e^{j \omega_{1}}\right) \cdot \nabla \nabla \nabla$

Oblique Projections: Some of the results in this section can also be found in the mathematics literature, in the more general setting of oblique projections [2]-[5]. The article by Aldroubi and Unser [3] is especially insightful for the discrete-time case. See [3, Ths. 1 and 2] to see the connection to this section. The papers by Cohen et al. [4], [5] address many deep issues in the continuous-time case.

\section{APPLICATION IN LEAST SQUARES INTERPOLATION}

Consider the class $\mathcal{F}$ of discrete time signals $y(n)$ that can be modeled as the output of a fixed interpolation filter $F(z)$, as shown in Fig. 9. We will refer to this as the interpolation-filter model. One situation where this model arises is in sampling theory. We can reconstruct $y(n)$ from the decimated version $y(n M)$ under some mild conditions [25], [26]. Given an arbitrary signal $x(n) \in \ell_{2}$, suppose we wish to approximate it by the model signal $y(n) \in \mathcal{F}$. This can be done by proper choice of the lower rate signal $c(n)$. Let us say we want $c(n)$ to be chosen such that

$$
\sum_{n}|y(n)-x(n)|^{2}
$$

is minimized. This least squares solution $y(n)$ is nothing but the orthogonal projection of $x(n)$ onto $\mathcal{F}$. This problem is related to a number of things in filterbank theory, as we will see. In particular, it arises in the context of least square spline approximation of continuous time $L_{2}$ signals as shown by Unser et al. [20]. It also arises in the optimal subband coder problem as shown by Strintzis; see, for example, [14].

Theorem 4: Given the filter $F(z)$, assume $\left(\left|F\left(e^{j \omega}\right)\right|^{2}\right)_{\downarrow M}>$ 0 , and define a new filter

$$
H(z)=\frac{\tilde{F}(z)}{[\tilde{F}(z) F(z)]_{\downarrow M \uparrow M}} .
$$

If we pass the given signal $x(n)$ through $H(z)$ and decimate it by $M$, we get the optimal $c(n)$ [see Fig. 10(a)]. This $c(n)$ can be used to find the least squares approximation $y(n)$. The complete system is shown in Fig. 10(b). The filter (6) is called the

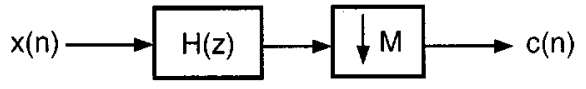

(a)

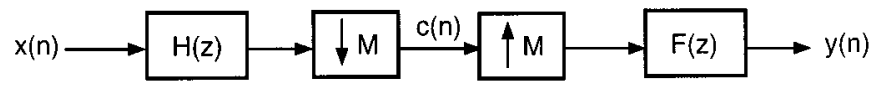

(b)

Fig. 10. Generating the best approximation $y(n)$ of the given signal $x(n)$. (a) Computing the driving signal $c(n)$. (b) Complete system.

(orthogonal) projection prefilter corresponding to the interpolation filter $F(z)$.

Proof: The assumption $\left(\left|F\left(e^{j \omega}\right)\right|^{2}\right)_{\downarrow M}>0$ ensures that the denominator of $H(z)$ does not have unit circle zeros. The squared error can be rewritten in the frequency domain as follows:

$$
\begin{aligned}
& \sum_{n}|y(n)-x(n)|^{2} \\
& =\int_{0}^{2 \pi}\left|Y\left(e^{j \omega}\right)-X\left(e^{j \omega}\right)\right|^{2} d \omega / 2 \pi \\
& =\int_{0}^{2 \pi}\left|C\left(e^{j \omega M}\right) F\left(e^{j \omega}\right)-X\left(e^{j \omega}\right)\right|^{2} d \omega / 2 \pi .
\end{aligned}
$$

The aim is to choose $C\left(e^{j \omega}\right)$ optimally to minimize this. Notice that $C\left(e^{j \omega M}\right)$ appearing in the integrand has period $2 \pi / M$ and can be chosen independently only in the range $0 \leq \omega<2 \pi / M$. Therefore, let us rewrite

$$
\begin{aligned}
\sum_{n} \mid & y(n)-\left.x(n)\right|^{2} \\
= & \int_{0}^{2 \pi / M} \\
& \times \underbrace{\sum_{k=0}^{M-1}\left|C\left(e^{j \omega M}\right) F\left(e^{j(\omega+2 \pi k / M)}\right)-X\left(e^{j(\omega+2 \pi k / M)}\right)\right|^{2}}_{Q(\omega)} \\
& \times d \omega / 2 \pi .
\end{aligned}
$$

For each $\omega$ in $0 \leq \omega<2 \pi / M$, we can choose $C\left(e^{j \omega M}\right)$ independently such that the non-negative integrand $Q(\omega)$ is minimized. Note that $C\left(e^{j \omega M}\right)$ is independent of the summation index $k$. Define the vectors

$$
\begin{aligned}
& \mathbf{a}(\omega)=\left[\begin{array}{c}
X\left(e^{j \omega}\right) \\
X\left(e^{j(\omega+2 \pi / M)}\right) \\
\vdots \\
X\left(e^{j(\omega+(2 \pi(M-1)) / M)}\right)
\end{array}\right] \\
& \mathbf{b}(\omega)=\left[\begin{array}{c}
F\left(e^{j \omega}\right) \\
F\left(e^{j(\omega+2 \pi / M)}\right) \\
\vdots \\
F\left(e^{j(\omega+(2 \pi(M-1)) / M)}\right)
\end{array}\right] .
\end{aligned}
$$

The problem is that of minimizing

$$
\begin{aligned}
\left\|C\left(e^{j \omega M}\right) \mathbf{b}(\omega)-\mathbf{a}(\omega)\right\|^{2} \\
=C^{*}\left(e^{j \omega M}\right) \mathbf{b}^{\dagger}(\omega) \mathbf{b}(\omega) C\left(e^{j \omega M}\right)-C^{*}\left(e^{j \omega M}\right) \mathbf{b}^{\dagger}(\omega) \mathbf{a}(\omega) \\
\quad-\mathbf{a}^{\dagger}(\omega) \mathbf{b}(\omega) C\left(e^{j \omega M}\right)+\mathbf{a}^{\dagger}(\omega) \mathbf{a}(\omega)
\end{aligned}
$$


By using the familiar trick of "completion of squares," this can be rewritten as

$$
\begin{aligned}
\left\|C\left(e^{j \omega M}\right) \mathbf{b}(\omega)-\mathbf{a}(\omega)\right\|^{2} \\
=\left(C^{*}\left(e^{j \omega M}\right)-\frac{\mathbf{a}^{\dagger} \mathbf{b}}{\mathbf{b}^{\dagger} \mathbf{b}}\right) \mathbf{b}^{\dagger} \mathbf{b}\left(C\left(e^{j \omega M}\right)-\frac{\mathbf{b}^{\dagger} \mathbf{a}}{\mathbf{b}^{\dagger} \mathbf{b}}\right) \\
\quad+\mathbf{a}^{\dagger} \mathbf{a}-\frac{\left|\mathbf{a}^{\dagger} \mathbf{b}\right|^{2}}{\mathbf{b}^{\dagger} \mathbf{b}}
\end{aligned}
$$

This shows that the best unique choice of $C\left(e^{j \omega M}\right)$ is $C\left(e^{j \omega M}\right)=\mathbf{b}^{\dagger}(\omega) \mathbf{a}(\omega) / \mathbf{b}^{\dagger}(\omega) \mathbf{b}(\omega)$. To rewrite this in terms of filters and multirate building blocks, recall that [23] $\left[S\left(e^{j \omega}\right)\right]_{\downarrow M}=\sum_{k=0}^{M-1} S\left(e^{j(\omega+2 \pi k) / M}\right) / M$. Thus

$$
\begin{aligned}
\mathbf{b}^{\dagger}(\omega) \mathbf{a}(\omega) & =\sum_{k=0}^{M-1} F^{*}\left(e^{j(\omega+2 \pi k / M)}\right) X\left(e^{j(\omega+2 \pi k / M)}\right) \\
& =M\left[F^{*}\left(e^{j \omega}\right) X\left(e^{j \omega}\right)\right]_{\downarrow M \uparrow M} \\
\mathbf{b}^{\dagger}(\omega) \mathbf{b}(\omega) & =\sum_{k=0}^{M-1}\left|F\left(e^{j(\omega+2 \pi k / M)}\right)\right|^{2} \\
& =M\left[\left|F\left(e^{j \omega}\right)\right|^{2}\right]_{\downarrow M \uparrow M} .
\end{aligned}
$$

The optimal $C\left(e^{j \omega M}\right)$ is therefore $C\left(e^{j \omega M}\right)=\left(\left[F^{*}\left(e^{j \omega}\right)\right.\right.$ $\left.\left.X\left(e^{j \omega}\right)\right]_{\downarrow M \uparrow M}\right) /\left(\left[\left|F\left(e^{j \omega}\right)\right|^{2}\right]_{\downarrow M \uparrow M}\right)$. That is

$$
\begin{aligned}
C(z) & =\frac{[\tilde{F}(z) X(z)]_{\downarrow M}}{[\tilde{F}(z) F(z)]_{\downarrow M}} \\
& =[\underbrace{\left(\frac{\tilde{F}(z)}{[\widetilde{F}(z) F(z)]_{\downarrow M \uparrow M}}\right)}_{H(z)} X(z)]_{\downarrow M} .
\end{aligned}
$$

That is, $c(n)$ can be generated by filtering $x(n)$ through $H(z)$, and decimating by $M$ [see Fig. 10(b)].

$\nabla \nabla \nabla$

Theorem 5-Uniqueness of Prefilter: For fixed $F(z)$ and $x(n) \in \ell_{2}$, the least squares approximation $y(n)$ is unique, and so is the generating signal $c(n)$. Next, suppose the prefilter $H(z)$ is such that the output of $F(z)$ is the least squares approximation of $x(n)$ for any choice of the $\ell_{2}$ input $x(n)$ in Fig. 10(b). Then, $H(z)$ is unique and is therefore given by the projection prefilter (6).

Proof: The uniqueness of $y(n)$ and $c(n)$ follows from the proof of Theorem 4. Next, let $H(z)$ and $H_{1}(z)$ be two prefilters, and let them both be optimal for all $x(n) \in \ell_{2}$. Since the optimal $c(n)$ is unique as seen from the proof of Theorem 4 , we see that

$$
\left(X(z)\left[H(z)-H_{1}(z)\right]\right)_{\downarrow M}=0
$$

for all $X(z)$. Choosing $X(z)=z^{k}$, this implies that the $k$ th polyphase component of $H(z)-H_{1}(z)$ is zero [23]. Since this is true for all $k$, we conclude $H(z)-H_{1}(z)=0$. Therefore, the prefilter is unique. Although this argument is elegant, the result of Theorem 5 also follows from the uniqueness of the orthogonal projection operator onto a closed subspace [3].

\section{Remarks:}

1) Partner Property: Note that $H(z)$ given by (6) is a biorthogonal partner of $F(z)$, that is, $[H(z) F(z)]_{\downarrow M}$ $=1$. This follows from Theorem 1 by setting $G(z)=\tilde{F}(z)$. The assumption $\left(\left|F\left(e^{j \omega}\right)\right|^{2}\right)_{\downarrow M}>0$ in Theorem 4 is equivalent to the statement that a biorthogonal partner exists (Theorem 3). Even though the optimal prefilter $H(z)$ generating $y(n)$ is a biorthogonal partner of $F(z)$, we see from Theorem 5 that an arbitrary biorthogonal partner of $F(z)$ will not work in the least squares problem. This is unlike in Section III, where the signal $c(n)$ could be produced from $x(n)$ using any biorthogonal partner $H(z)$.

2) Orthonormal Case: If $(\tilde{F}(z) F(z))_{\downarrow M}=1$, that is, $\tilde{F}(z) F(z)$ is Nyquist $(M)$, then the solution for $H(z)$ becomes $H(z)=\tilde{F}(z)$, that is, $h(n)=f^{*}(-n)$, which is time-reversed conjugation, as in matched filtering. Recall that this condition arises in each subband of an orthonormal filterbank. Indeed, the interpolated subband outputs in any orthonormal filterbank represent projections of the input $x(n)$ onto subspaces spanned by the synthesis filter functions $\eta_{k i}(n)=f_{i}(n-M k)$.

Example 2: We now present an example demonstrating various aspects of the least squares interpolation model. Let $M=$ 2 , and assume $F(z)$ is the first-order FIR filter $F(z)=1+b z^{-1}$ for some real $b$. Then, $(\tilde{F}(z) F(z))_{\downarrow 2}=1+b^{2}$, and Theorem 4 yields

$$
H(z)=\frac{\tilde{F}(z)}{[\tilde{F}(z) F(z)]_{\downarrow 2 \uparrow 2}}=\frac{1+b z}{1+b^{2}} .
$$

Suppose we wish to approximate a finite-duration signal $x(n)$ with $z$-transform $X(z)=1+a z^{-1}$. Then, the optimal $C(z)$ is given by

$$
\begin{aligned}
C(z) & =(X(z) H(z))_{\downarrow 2} \\
& =\left(\frac{1+a b+a z^{-1}+b z}{1+b^{2}}\right)_{\downarrow 2} \\
& =\frac{1+a b}{1+b^{2}} .
\end{aligned}
$$

To demonstrate that arbitrary biorthogonal partners may not be optimal, consider the biorthogonal partner of $F(z)$ given by $H_{2}(z)=1 / F(z)=1 /\left(1+b z^{-1}\right)$. The decimated subband signal is $\left[X(z) H_{2}(z)\right]_{\downarrow 2}=\left(1-a b z^{-1}\right) /\left(1-b^{2} z^{-1}\right)$ and is not the optimal $C(z)$. Consider next a transfer function of the form $E(z)=z^{-2 K}(1-z / a)$, where $K$ is an arbitrary integer. We have

$$
(X(z) E(z))_{\downarrow 2}=0
$$

which shows that if $H(z)$ is replaced with $H_{1}(z)=H(z)+$ $E(z)$, then the output of $F(z)$ in Fig. 10(b) is still the least squares approximation $y(n)$. This shows that the optimum prefilter is not unique; in fact, $H_{1}(z)$ is not even a biorthogonal partner. These instances occur if the goal is to make the prefilter work for only some specific choices of $x(n)$. If the prefilter has to work for all $x(n) \in \ell_{2}$, then (6) is the only choice. 
Summary of This Section: Here is the summary of what we have shown under the mild assumption that $\left(\left|F\left(e^{j \omega}\right)\right|^{2}\right)_{\downarrow M}>0$. The least squares approximation $y(n)$ is unique, and so is the driving signal $c(n)$. If the prefilter $H(z)$ has to generate the optimal $c(n)$ for all $x(n) \in \ell_{2}$, then $H(z)$ is the unique filter called the projection prefilter and is given by (6). This prefilter also happens to be a biorthogonal partner of $F(z)$.

\section{Continuous-Time InTERPOLATION MODELS}

We now show how the results of earlier sections find application in interpolation based on continuous-time models. As a first step, we review a well-known linear interpolation model and its efficient implementation developed in the fundamental work by Unser et al. [19]. An excellent review of sampling in this context was recently given by Unser [22].

\section{A. Review}

Given a discrete time signal $x_{d}(n)$ and an arbitrary function $\phi(t)$, we can almost always assume that $x_{d}(n)$ can be written in the form

$$
x_{d}(n)=\sum_{k=-\infty}^{\infty} c(k) \phi(n-k)
$$

for appropriate choice of $c(k)$. This is because the equation is equivalent to $X_{d}\left(e^{j \omega}\right)=C\left(e^{j \omega}\right) \Phi_{d, 1}\left(e^{j \omega}\right)$ in the frequency domain, where $\Phi_{d, 1}\left(e^{j \omega}\right)$ is the discrete-time FT of the sampled sequence $\phi(n)$ (the need for a second subscript " 1 " will be clear soon). Thus, we can calculate $c(k)$ from $x_{d}(n)$ by inverse digital filtering, that is, $C\left(e^{j \omega}\right)=X_{d}\left(e^{j \omega}\right) / \Phi_{d, 1}\left(e^{j \omega}\right)$. The only theoretical condition is that $\Phi_{d, 1}\left(e^{j \omega}\right)$ be nonzero for all $\omega$ so that $1 / \Phi_{d, 1}\left(e^{j \omega}\right)$ represents a stable filter. As we will see, the stability condition can readily be satisfied in practice.

The preceding observation shows that we can regard $x_{d}(n)$ as samples of a continuous-time signal $x(t)$, which admits the specific model

$$
x(t)=\sum_{k=-\infty}^{\infty} c(k) \phi(t-k)
$$

where the sample spacing is $T=1$. While true for almost any $\phi(t)$, this is especially useful for certain choices of $\phi(t)$. For example, if $\phi(t)$ has smoothness properties such as a certain degree of differentiability everywhere, then we can use this to generate a good interpolated version of $x(n)$. A $256 \times 256$ image can be displayed as a $512 \times 512$ image in this way (interpolation by two). Smoothness of $\phi(t)$ usually ensures that the interpolated result is visually pleasing (see example below). To see how the model can be used for interpolation, notice that the samples of $x(t)$ at a finer spacing $1 / L$ are given by

$$
\begin{aligned}
x(n / L) & =\sum_{k=-\infty}^{\infty} c(k) \phi(n / L-k) \\
& =\sum_{k=-\infty}^{\infty} c(k) \phi\left(\frac{n-k L}{L}\right) \\
& =\sum_{k=-\infty}^{\infty} c(k) \phi_{d, L}(n-k L)
\end{aligned}
$$

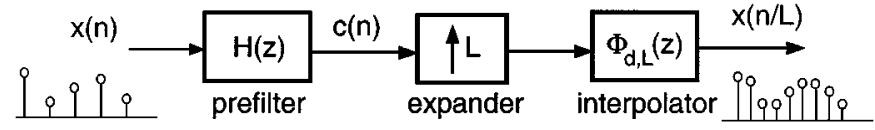

Fig. 11. Interpolation of a signal $x(n)$ with digital filters. The signal is assumed to have a continuous-time model $x(t)=\sum_{k} c(k) \phi(t-k)$.

where $\phi_{d, L}(n)=\phi(n / L)$ is the filter obtained by sampling $\phi(t)$ at a finer spacing of $1 / L$. In summary, we can reconstruct the finer samples $x(n / L)$ from $x(n)$, as shown in Fig. 11. We first pass $x(n)$ through the digital prefilter

$$
H\left(e^{j \omega}\right)=1 / \Phi_{d, 1}\left(e^{j \omega}\right) .
$$

This gives $c(n)$. Then, we use the $L$-fold upsampler or expander [23], followed by the interpolation filter $\Phi_{d, L}(z)=\sum_{n} \phi_{d, L}(n) z^{-n}$. We see that the interpolation from $x(n)$ to $x(n / L)$ can be done entirely digitally. The function $\phi(t)$ is often chosen as a spline function, where the use of cubic splines is especially common. For the rest of the section, we will frequently use the following notations:

$$
\phi_{d, L}(n) \triangleq \phi(n / L), \quad \Phi_{d, L}(z)=\sum_{n} \phi_{d, L}(n) z^{-n} .
$$

In many practical systems, the function $\phi(t)$ is of finite duration. This makes $\phi_{d, 1}(n)$ FIR, which means that $H\left(e^{j \omega}\right)=$ $1 / \Phi_{d, 1}\left(e^{j \omega}\right)$ is IIR. In general, this IIR filter may not have all poles inside the unit circle (this problem arises when $\phi(t)$ is a $B$-spline [19]). An $N$ th order $B$-spline is nothing but the convolution of the pulse function

$$
p(t)= \begin{cases}1, & 0 \leq t<1 \\ 0, & \text { otherwise }\end{cases}
$$

with itself $N$ times so that the Fourier transform of the $N$ th-order $B$-spline is ${ }^{4}$

$$
\Phi(j \omega)=e^{-j \omega(N+1) / 2}\left(\frac{\sin (\omega / 2)}{(\omega / 2)}\right)^{N+1} .
$$

It can be verified [13] that the corresponding time domain expression is

$$
\phi(t)=\sum_{k=0}^{N+1}\left(\begin{array}{c}
N+1 \\
k
\end{array}\right)(-1)^{k} \frac{(t-k)^{N}}{N !} \mathcal{U}(t-k)
$$

where $\mathcal{U}(t)$ is the unit step function. The beauty of an $N$ th-order spline is that it is continuously differentiable $N-1$ times everywhere [i.e., the $(N-1)$ th derivative exists and is continuous]. Moreover, the $N$ th derivative is a piecewise constant. The differentiability is true even at the end points for finite-duration splines such as the $B$-spline, which has duration $N+1$. In fact, $N$ th-order splines are polynomials of degree $N$ between integers. These polynomial pieces are glued together such that they are sufficiently differentiable even at the integers.

For example, assume that $\phi(t)$ is the third-order $B$-spline (or cubic spline), which is popularly used in image interpolation [19]. In this case, it can be shown that the sampled version $\phi(n)$

\footnotetext{
${ }^{4}$ Note that the $\Phi(j \omega)$ decays as $1 / \omega^{N+1}$. In some papers, this decay rate $(N+1)$ is regarded as the spline order.
} 


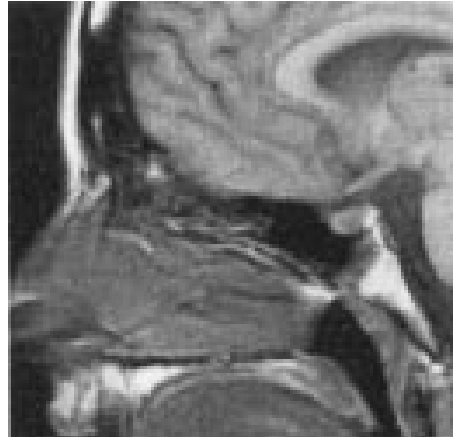

Fig. 12. A $128 \times 128$ region of the Eve image.

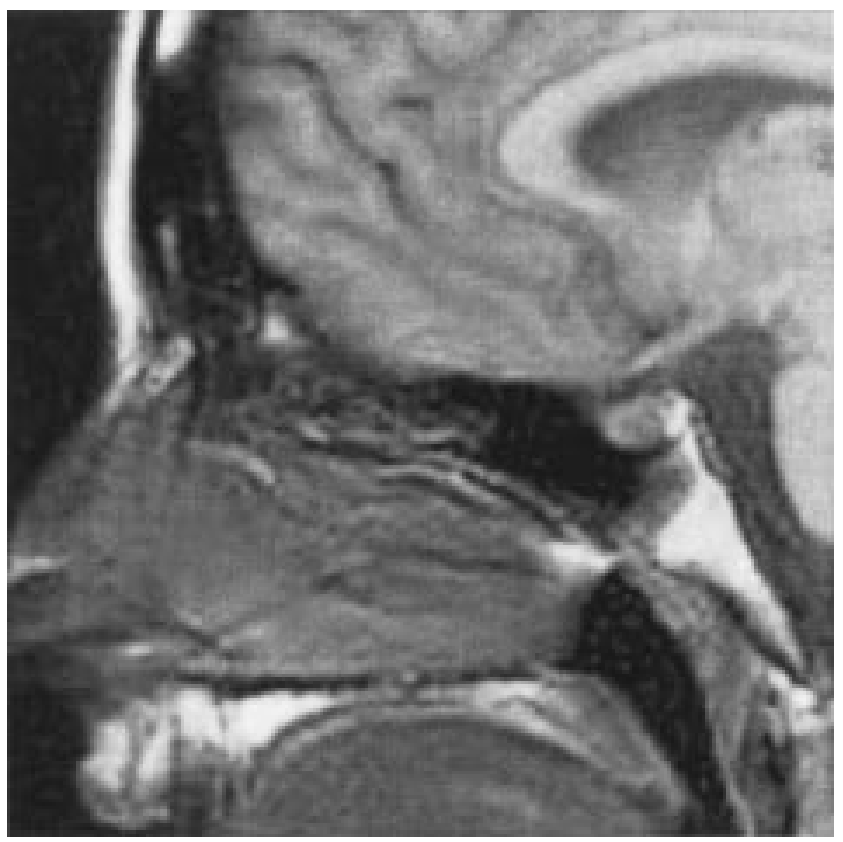

Fig. 13. A $256 \times 256$ interpolated version of the Eve image of Fig. 12, using the structure of Fig. 11, where $\phi(t)$ is the cubic spline.

has a $z$-transform $\Phi_{d, 1}(z)=\left(z^{-1}+4 z^{-2}+z^{-3}\right) / 6$, which is FIR. Therefore, the prefilter $H(z)$ is the allpole filter given by

$$
H(z)=\frac{6 z}{1+4 z^{-1}+z^{-2}} .
$$

The denominator is a symmetric polynomial, which shows that at least one pole has magnitude $\geq 1$. Indeed, the poles are -3.7321 and -0.2679 . This shows that there is no causal stable implementation. Efficient noncausal implementations that make the spline interpolation very practical are described in [19]. The spline interpolation filter $\Phi_{d, L}(z)$ for $L=2$ is given by

$$
\begin{aligned}
\Phi_{d, 2}(z)=\frac{z^{-1}}{48}( & +8 z^{-1}+23 z^{-2}+32 z^{-3} \\
& \left.+23 z^{-4}+8 z^{-5}+z^{-6}\right) .
\end{aligned}
$$

Fig. 12 shows a $128 \times 128$ portion of the Eve image, and Fig. 13 shows the two-fold interpolated version $(256 \times 256)$ obtained by using the above filters $H(z)$ and $\Phi_{d, 2}(z)$ in Fig. 11.

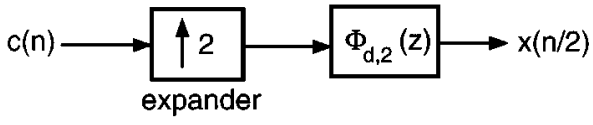

Fig. 14. Interpreting $x(n / 2)$ as the output of an interpolation filter $\Phi_{d, 2}(z)$, where $\phi_{d, 2}(n)=\phi(n / 2)$.

\section{B. All-FIR Interpolation}

Consider again the signal model $x(t)=\sum_{k=-\infty}^{\infty} c(k) \phi(t-$ $k$ ), but assume that we are given the samples at the finer spacing $T=1 / 2$. That is, we are given the oversampled version

$$
x(n / 2)=\sum_{k=-\infty}^{\infty} c(k) \phi\left(\frac{n}{2}-k\right) .
$$

In this case, we can often find the interpolated samples $x(n / K)$ for any $K$ using only FIR filters. To see this, let $\phi_{d, 2}(n)=\phi(n / 2)$ as usual, and rewrite the preceding as $x(n / 2)=\sum_{k=-\infty}^{\infty} c(k) \phi_{d, 2}(n-2 k)$. This shows that $x(n / 2)$ is the output of a digital interpolation filter, as shown in Fig. 14. If $\Phi_{d, 2}(z)$ is FIR and satisfies the conditions of Theorem 2 (with $M=2$ ), then it has an FIR biorthogonal partner $H(z)$ to recover $c(n)$ from $x(n / 2)$. Once we have $c(n)$, we can compute $x(t)$ for any $t$, for example, we can compute it at the finer spacing $1 / K$ by observing that

$$
\begin{aligned}
x(n / K) & =\sum_{k=-\infty}^{\infty} c(k) \phi\left(\frac{n}{K}-k\right) \\
& =\sum_{k=-\infty}^{\infty} c(k) \phi_{d, K}(n-K k) .
\end{aligned}
$$

Thus, we get the interpolation scheme shown in Fig. 15, where $H(z)$ and $\Phi_{d, K}(z)$ are both FIR.

Summary: Suppose a discrete-time signal $x_{d}(n)$ can be modeled as $x_{d}(n)=x(n / 2)$, where $x(t)$ is a continuous-time signal modeled as $x(t)=\sum_{k} c(k) \phi(t-k)$. That is, $x_{d}(n)$ is an oversampled version of $x(n)$ with an oversampling factor of two. Then, we can recover the samples at finer spacings such as $x(n / K)$ by using the multirate system shown in Fig. 15. If $\phi(t)$ has finite duration, then $\Phi_{d, K}(z)$ is FIR. If the two polyphase components of $\Phi_{d, 2}(z)$ do not have common zeros, then the filter $H(z)$ can be chosen to be FIR as well. Finally, we would like to point out that it is possible to compute $c(n)$ using FIR filters even without oversampling of any kind. The trick is to use nonuniform sampling, as shown in [8].

Generalization: If $x_{d}(n)=x(n / M)=\sum_{k=-\infty}^{\infty} c(k)$ $\phi(n / M-k)$, then we can represent it as in Fig. 16(a), where $\Phi_{d, M}(z)$ is a digital filter with impulse response $\phi_{d, M}(n) \triangleq \phi(n / M)$. This is an FIR filter if $\phi(t)$ has finite duration. If there is no common zero shared by all the $M$ polyphase components of $\Phi_{d, M}(z)$, then according to Theorem 2, there exists an FIR filter $H(z)$ such that $c(n)$ can be recovered from $x(n / M)$, as shown in Fig. 16(b). Thus, we can obtain interpolated versions $x(n / K)$ for any $K>M$ using the structure of Fig. 16(b). In fact, we can even take $K<M$, which yields fractional decimation by $M / K$. 


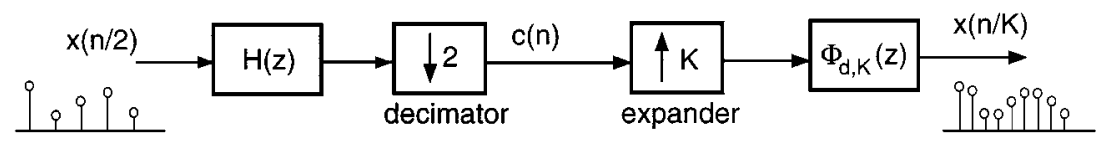

Fig. 15. Interpolation of a signal $x(n / 2)$ with digital filters. The signal is assumed to have a continuous time model $x(t)=\sum_{k} c(k) \phi(t-k)$. It is possible to make $H(z)$ FIR for finite-duration $\phi(t)$.

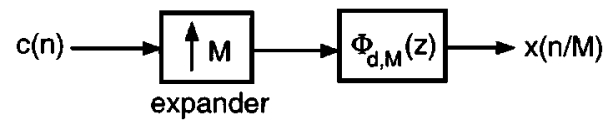

(a)

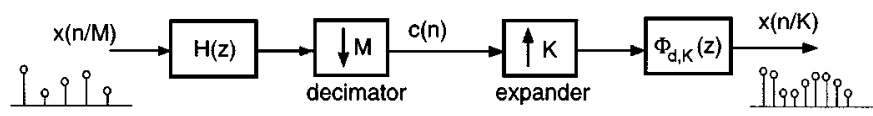

(b)

Fig. 16. (a) Model for $x(n / M)$, and (b) further interpolation of $x(n / M)$ with digital filters. The underlying continuous time model is $x(t)=\sum c(k) \phi(t-$ $k)$. It is possible to make $H(z)$ FIR for finite-duration $\phi(t)$.

Example 3: For example, assume again that $\phi(t)$ is the cubic spline. In this case

$$
\begin{aligned}
\Phi_{d, 2}(z)=\frac{z^{-1}}{48}( & +8 z^{-1}+23 z^{-2}+32 z^{-3} \\
& \left.+23 z^{-4}+8 z^{-5}+z^{-6}\right) .
\end{aligned}
$$

This can be written in the polyphase form $\Phi_{d, 2}(z)=R_{0}\left(z^{2}\right)+$ $z R_{1}\left(z^{2}\right)$, where

$$
\begin{aligned}
& R_{0}(z)=\frac{8 z^{-1}+32 z^{-2}+8 z^{-3}}{48} \\
& R_{1}(z)=\frac{z^{-1}+23 z^{-2}+23 z^{-3}+z^{-4}}{48} .
\end{aligned}
$$

These polynomials are coprime. This can be verified either by running Euclid's algorithm or by explicit computation of their zeros [the finite zeros of $R_{0}(z)$ are -3.73 and -0.268 , whereas those of $R_{1}(z)$ are $-21.95,-1$ and -0.0455 , showing that these are coprime]. Therefore, there exist FIR filters $E_{0}(z)$ and $E_{1}(z)$ such that $E_{0}(z) R_{0}(z)+E_{1}(z) R_{1}(z)=1$. Indeed, the pair

$$
E_{0}(z)=\frac{3 z^{5}+68 z^{4}+47 z^{3}}{6}, \quad E_{1}(z)=\frac{-24 z^{5}-88 z^{4}}{6}
$$

yields $E_{0}(z) R_{0}(z)+E_{1}(z) R_{1}(z)=1$. The FIR filter $H(z)$ in Fig. 15 is therefore

$$
\begin{aligned}
H(z) & =E_{0}\left(z^{2}\right)+z^{-1} E_{1}\left(z^{2}\right) \\
& =\frac{3 z^{10}-24 z^{9}+68 z^{8}-88 z^{7}+47 z^{6}}{6} .
\end{aligned}
$$

To demonstrate, we consider the $256 \times 256$ oversampled image of Eve shown in Fig. 13. Then, we can model it satisfactorily as $x(n / 2)=\sum_{k=-\infty}^{\infty} c(k) \phi(n / 2-k)$, where $\phi(t)$ is the cubic spline. Suppose we want to interpolate this into a $512 \times 512$ image. Then, we can do it using the scheme of Fig. 15, where $H(z)$ and $\phi_{d, 4}(z)$ are FIR filters. The result of interpolation is shown in Fig. 17. For comparison, Fig. 18 shows the result of direct four-fold interpolation of the $128 \times 128$ section using the standard noncausal IIR filter method [19].

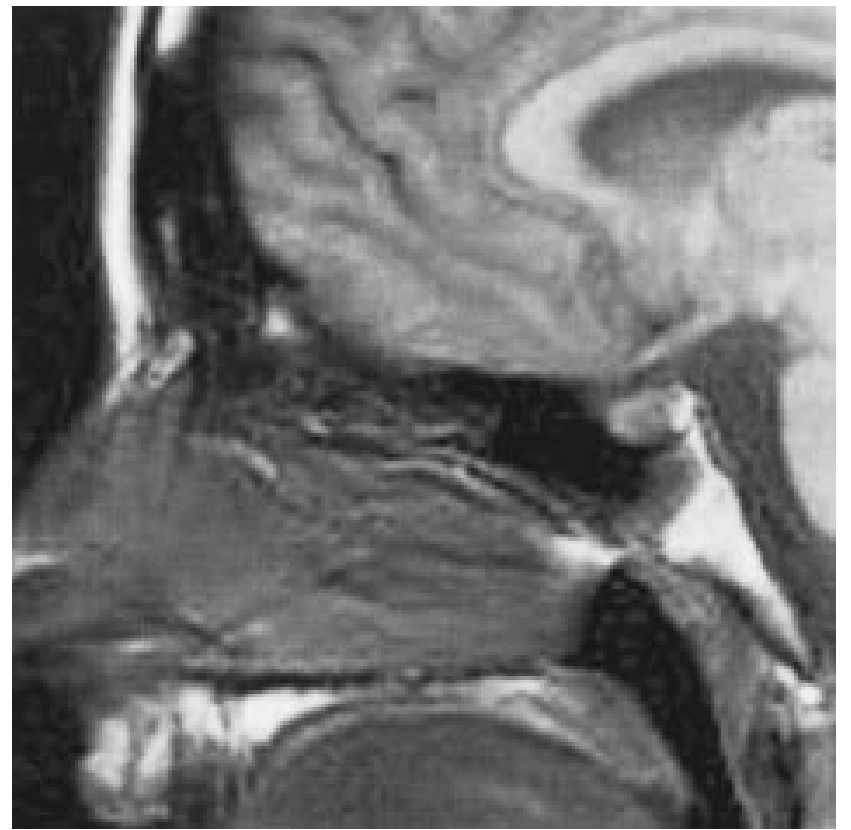

Fig. 17. FIR based two-fold cubic-spline interpolation of the $256 \times 256$ Eve image shown in Fig. 13.

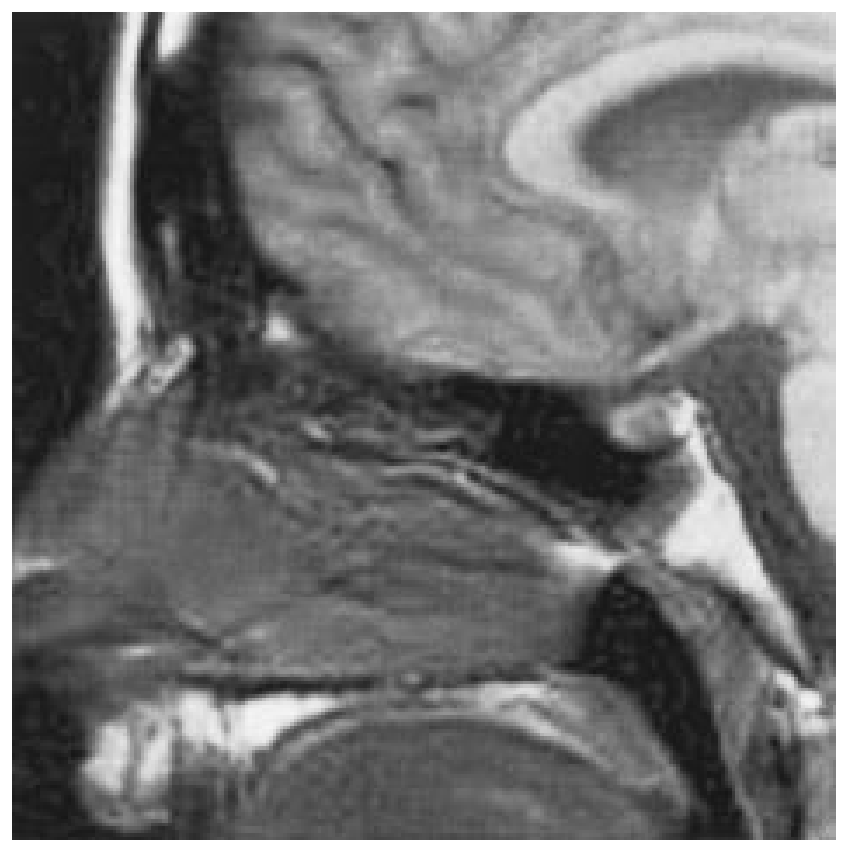

Fig. 18. Direct four-fold cubic-spline interpolation of the $128 \times 128$ region of Eve image using traditional IIR method [19].

\section{Application in Multiresolution Theory}

For signals of the form $x(t)=\sum_{k} c(k) \phi(t-k)$, where $\phi(t)$ is a fixed finite duration function, if we only have the samples $x(n)$, then we need the IIR filter $1 / \Phi_{d}\left(e^{j \omega}\right)$ to compute $c(k)$. We also just showed that if the oversampled version 
$x(n / 2)$ is available, then we can compute $c(k)$ using only FIR filters. This is an attractive alternative to what is conventionally done in multiresolution analysis ${ }^{5}$ to compute $c(k)$ from a highly oversampled version. To appreciate the difference between the above FIR construction and the conventional construction, we now give a brief review of the latter. Assuming $c(k)$ is in $\ell_{2}$ and $\phi(t) \in L^{2}$, the set of functions $x(t)=\sum_{k} c(k) \phi(t-k)$ forms a subspace $V_{0} \subset L^{2}$. This subspace is spanned by the integer-shifted versions $\{\phi(t-k)\}$ [see Fig. 19(a)].

Now, consider the squeezed version $\phi(2 t)$ and its shifted versions $\phi(2 t-k)=\phi(2(t-0.5 k))$ sketched in Fig. 19(b). This set $\{\phi(2(t-0.5 k))\}$ also spans a subspace $V_{1} \subset L_{2}$. In multiresolution theory, $\phi(t)$ is chosen such that $V_{0} \subset V_{1}$. In particular, $\phi(t)$ is a linear combination of $\phi(2 t-k)$, that is

$$
\phi(t)=2 \sum_{m} f(m) \phi(2 t-m) .
$$

This is the familiar dilation equation [6], [10] and translates in the Fourier domain to $\Phi(j \omega)=F\left(e^{j \omega / 2}\right) \Phi(j \omega / 2)$. By repeating this idea, we see that $x(t)$ belongs to the space $V_{J}$ spanned by $\left\{\phi\left(2^{J} t-k\right)\right\}$ for any integer $J \geq 0$, that is

$$
x(t)=\sum_{k} c_{J}(k) 2^{J / 2} \phi\left(2^{J} t-k\right) .
$$

The multiresolution coefficients $c_{J}(k)$ at scale $J$ reduce to the usual $c(k)$ for $J=0$. The constant $2^{J / 2}$ merely ensures that the scaled basis functions $\left\{2^{J / 2} \phi\left(2^{J} t-k\right)\right\}$ have the same energy for all $J$. Fig. 19(c) shows $x(t)$ and several shifted versions $\phi\left(2^{J} t-k\right)$. We will now argue that the samples $x\left(2^{-J} k\right)$ are approximately proportional to $c_{J}(k)$. Since $x\left(2^{-J} n\right)=\sum_{k} c_{J}(k) 2^{J / 2} \phi(n-k)$, the sequence $x\left(2^{-J} n\right)$ is the output of the digital filter $\phi(n)$ in response to the input $2^{J / 2} c_{J}(n)$. Thus, except for a constant multiplier, $c_{J}(n)$ is the output of the inverse filter $\gamma(n)$ in response to the input $x\left(2^{-J} n\right)$. If $J$ is large enough, then $x\left(2^{-J} n\right)$ is nearly constant in the region where $\gamma(n)$ is significant. Thus, the output $c_{J}(n)$ is also slowly varying and is nearly proportional to the input, that is, $c_{J}(k) \approx \alpha_{J} x\left(2^{-J} k\right)$. If the oversampling factor $2^{J}$ is large enough, this estimate of $c_{J}(k)$ is very good. The beauty of the dilation equation is that it allows us to compute the multiresolution coefficients at lower scales

$$
c_{J-1}(k), c_{J-2}(k), \ldots, c_{0}(k)
$$

successively from $c_{J}(k)$ and thereby identify $c(k)=c_{0}(k)$. A brief justification of this well-known result is given next for completeness.

Proof: Substituting the dilation equation (11) into the scale- $J$ representation (12), we get

$$
\begin{aligned}
x(t) & =\sqrt{2} \sum_{m} \sum_{k} c_{J}(k) f(m) 2^{(J+1) / 2} \phi\left(2^{J+1} t-2 k-m\right) \\
& =\sum_{\ell} \underbrace{\sum_{k} \sqrt{2} c_{J}(k) f(\ell-2 k)}_{c_{J+1}(\ell)} 2^{(J+1) / 2} \phi\left(2^{J+1} t-\ell\right) .
\end{aligned}
$$

${ }^{5}$ See Mallat's book [10] for an excellent treatment of multiresolution theory.

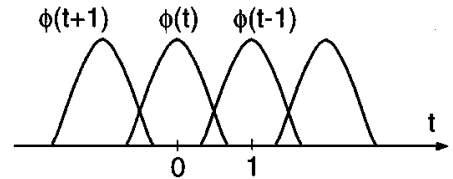

(a)

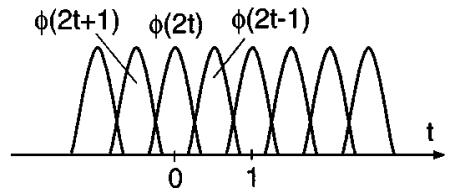

(b)

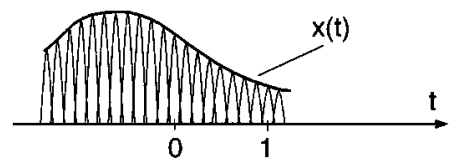

(c)

Fig. 19. (a) Function $\phi(t)$ and its integer shifted versions. (b) Squeezed function $\phi(2 t)$ and its shifted versions $\phi(2 t-k)$. (c) Several shifted and weighted versions $c_{J}(k) 2^{J / 2} \phi\left(2^{J} t-k\right)$ for $2^{J}=8$, shown along with $x(t)=\sum_{k} c_{J}(k) 2^{J / 2} \phi\left(2^{J} t-k\right)$.

That is, $c_{J+1}(n)=\sqrt{2} \sum_{k} c_{J}(k) f(n-2 k)$, which shows that we can go from scale $J$ multiresolution coefficients $c_{J}(n)$ to the scale $J+1$ coefficients $c_{J+1}(n)$ by using an interpolation filter as shown in Fig. 20(a), where $F(z)=\sum_{n} f(n) z^{-n}$. If $\sqrt{2} F(z)$ has a biorthogonal partner $\sqrt{2} H(z)$, we can also go from scale $J+1$ to $J$ by using the decimation filter of Fig. 20(b) (see Section II). This shows that we can compute the coefficients $c_{i}(n)$ for all lower scales using the multistage decimation system shown in Fig. 20(c).

$\nabla \nabla \nabla$

If $F(z)$ is FIR with coprime polyphase components $R_{0}(z)$ and $R_{1}(z)$ [where $F(z)=R_{0}\left(z^{2}\right)+z R_{1}\left(z^{2}\right)$ ], then we can find an FIR filter $H(z)$ to implement Fig. 20(c). Finally, notice that if $\left\{\eta_{k}(n)\right\}=\{\sqrt{2} f(n-2 k)\}$ is an orthonormal set, then $h(n)=f^{*}(-n)$ works in the preceding scheme (see Section II). In order for the above oversampling strategy to yield good results, we have to make the oversampling factor large so that the approximation of $c_{J}(n)$ is good. Compare this with the method of Section VI-B, which yields exact results and requires oversampling only by a factor of two, and the method in [8], which yields exact results with no oversampling at all (but uses nonuniform sampling).

\section{Fractionally SPaced EQualizers}

Consider the digital communication system shown in Fig. 21. Here, $x(n)$ represents a sequence of symbols with spacing $T$ seconds or symbol rate $1 / T \mathrm{~Hz}$. To be specific, assume that $x(n)$ can have $N$ possible amplitudes as in $N$-ary pulse amplitude modulation (PAM) [12]. The transmitting filter with impulse response $g_{c}(t)$ generates the continuous-time baseband signal $x_{c}(t)=\sum_{n} x(n) g_{c}(t-n T)$, which is then sent through the channel [subscript $c$ is used for continuous-time quantities, e.g., $x_{c}(t), X_{c}(j \omega)$, etc.]. The modulation step that imposes a carrier is ignored, as it does not affect our discussions. Assume that the channel can be modeled as a continuous-time LTI system with frequency response $C_{c}(j \omega)$, followed by an additive noise source as shown in the figure. The received signal $r_{c}(t)$ is then 


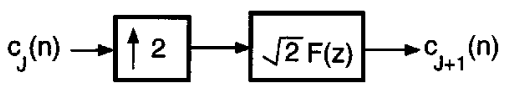

(a)

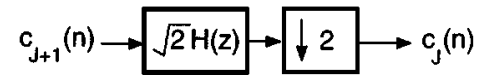

(b)

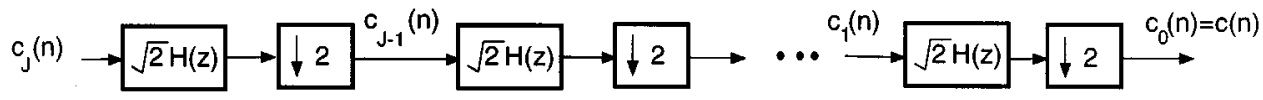

(c)

Fig. 20. Details of conventional multiresolution computation. (a) Representation of $c_{J+1}(n)$. (b) Computation of $c_{J}(n)$ from $c_{J+1}(n)$, where $\sqrt{2} H(z)$ is a biorthogonal partner of $\sqrt{2} F(z)$. (c) Multistage decimation circuit for computation of the coefficients $c_{i}(n)$ for all lower-level scales up to $c_{0}(n)=c(n)$.

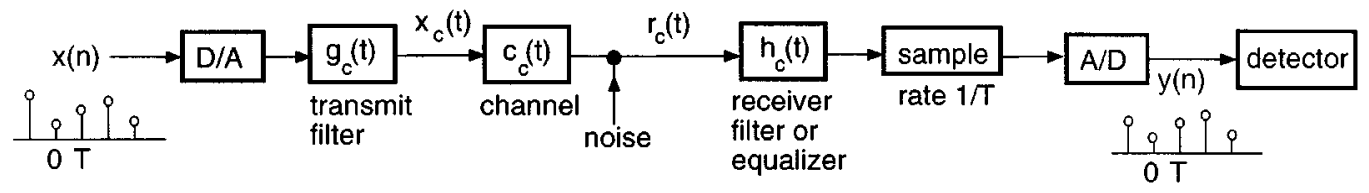

Fig. 21. Digital communication channel.

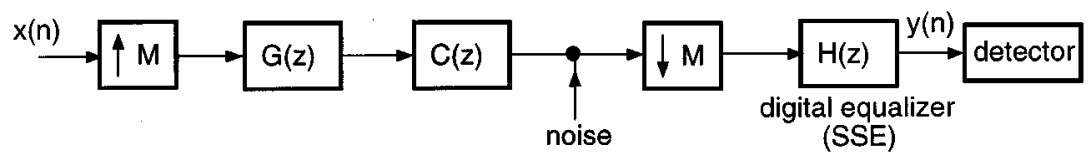

(a)

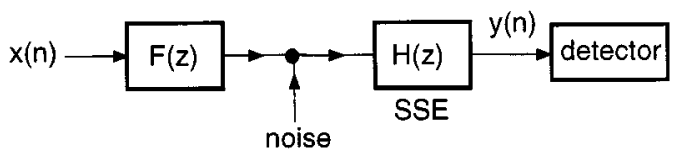

(b)

Fig. 22. (a) All-digital equivalent of the digital communication channel. (b) Further simplification where $F(z)=[G(z) C(z)]_{\downarrow M}$.

passed through a receiver filter $h_{c}(t)$ and sampled at the symbol rate $1 / T$. The resulting sequence $y(n)$ is input to the detector. Define

$$
F_{c}(j \omega) \triangleq G_{c}(j \omega) C_{c}(j \omega)
$$

The purpose of the receiver filter or equalizer $h_{c}(t)$ is to make sure that $F_{c}(j \omega) H_{c}(j \omega)$ has the Nyquist $(T)$ property (zero crossings at integer multiples of $T$ ) so that intersymbol interference (ISI) is avoided. In practice, the filter $g_{c}(t)$ can be implemented digitally before D/A conversion into the channel and the filter $h_{c}(t)$ implemented digitally after A/D conversion at the receiver. Assuming that the sampling at the receiver is done at the symbol rate $1 / T$, we obtain a digital equivalent of the entire system, as shown in Fig. 22(a). Here, $G(z)$ is an $M$-fold interpolation filter $[M$ is a large enough integer so that the pulse shape $g_{c}(t)$ is represented well by $\left.g(n)\right]$. The filter $C(z)$ represents the discrete time equivalent of $C_{c}(j \omega)$ sampled at $M$ times the symbol rate $1 / T$, and $H(z)$ is the digital filter representing the equalizer. Using the polyphase identity [23], this digital equivalent can be simplified as shown in Fig. 22(b), where $F(z)=[G(z) C(z)]_{\downarrow M}$. We say that $H(z)$ is the symbol-spaced equalizer (SSE) because it operates at the symbol rate $1 / T$. The discrete-time equivalent of the noise source can readily be identified and is not the main point of the discussion here. The filter $F(z)$ is often represented well with an FIR approximation. An ideal equalizer (or a zero-forcing equalizer [12]) has the form $H(z)=1 / F(z)$, which is IIR and typically of high order. In real-time implementations, the ideal equalizer is replaced with a practical FIR adaptive filter. It can be shown that the ISI suppression achieved by this filter is quite sensitive to the phase of sampling at the receiver [12]. The use of a so-called fractionally spaced equalizer (FSE) significantly reduces this problem and, moreover, allows FIR solutions [17].

To explain what an FSE is, consider again Fig. 21. Suppose the received signal is sampled at twice the symbol rate $2 / T$. We then use an equalizer $H_{2}(z)$ and downsample its output by 2 before sending it to the detector. The system can then be represented in discrete time multirate notation by Fig. 23(a) (assuming $M$ is even). This can be simplified into the form shown in Fig. 23(b), where $F_{2}(z)=[G(z) C(z)]_{\downarrow M / 2}$. (Again, the noise source can be adjusted accordingly). We see that the effective transfer function between the transmitted symbols $x(n)$ and received symbols is now $\left[F_{2}(z) H_{2}(z)\right]_{\downarrow 2}$. This can be made unity by designing $H_{2}(z)$ to be a biorthogonal partner of $F_{2}(z)$. The filter $H_{2}(z)$ is the fractionally spaced equalizer. It operates at twice the symbol rate. Typically, $F_{2}(z)$ represents an FIR approximation of $G_{c}(j \omega) C_{c}(j \omega)$. Thus, according to Theorem 2, it is almost always possible to find an FIR equalizer $H_{2}(z)$, the only mild condition being that the two polyphase components of $F_{2}(z)$ be coprime. The FSE technique not only offers an FIR solution, but it also reduces significantly the sensitivity of the 


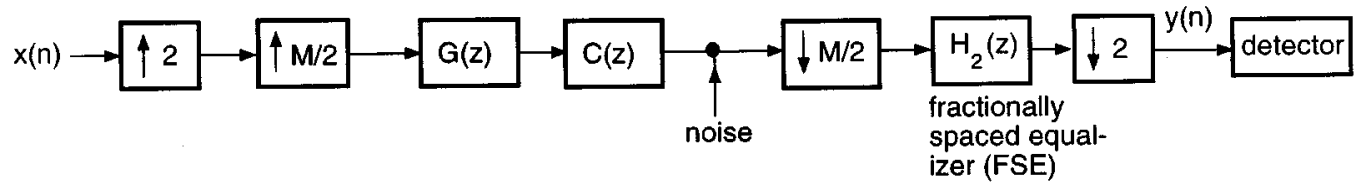

(a)

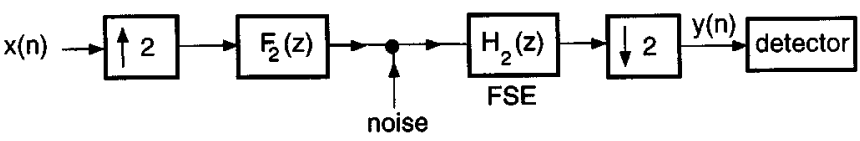

(b)

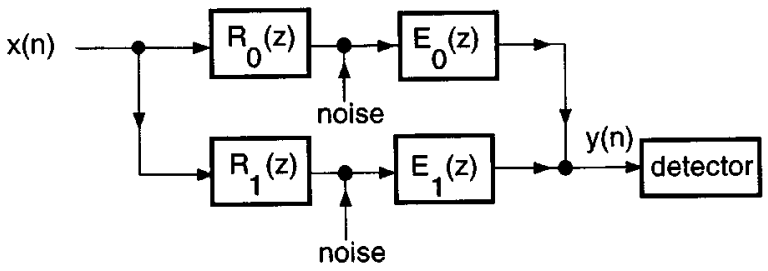

(c)

Fig. 23. Idea of a fractionally spaced equalizer (FSE). (a) Equalizer $H_{2}(z)$ operating at the rate $2 / T$. (b) Further simplification where $F_{2}(z)=[G(z) C(z)]_{\downarrow M / 2}$. (c) Polyphase representation.

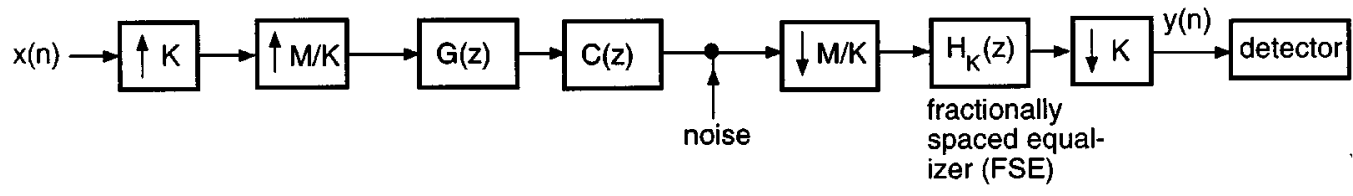

(a)

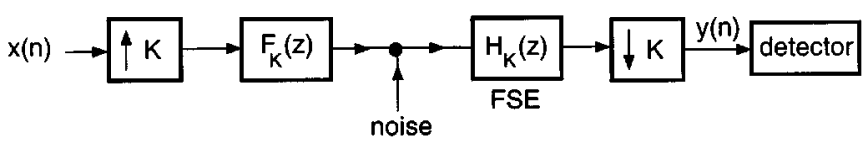

(b)

Fig. 24. Generalization of the fractionally spaced equalizer (FSE). (a) Equalizer $H_{K}(z)$ operating at the rate $K / T$. (b) Further simplification where $F_{K}(z)=$ $[G(z) C(z)]_{\downarrow M / K}$.

equalizer performance to errors in sampling phase, as demonstrated amply in [12].

Using the polyphase decompositions $H_{2}(z)=E_{0}\left(z^{2}\right)+$ $z^{-1} E_{1}\left(z^{2}\right)$ and $F_{2}(z)=R_{0}\left(z^{2}\right)+z R_{1}\left(z^{2}\right)$, we can redraw the structure of Fig. 23(b) as in Fig. 23(c), as shown in the proof of Theorem 2. Here, $R_{0}(z)$ represents the even samples of the impulse response of $F_{2}(z)$, and $R_{1}(z)$ represents the odd samples. Notice that $R_{0}(z)$ is precisely the quantity $F(z)$ in the symbol-spaced equalizer scheme of Fig. 22(b). Thus, the FSE structure takes into account the two interleaved sets of samples $R_{0}(z)$ and $R_{1}(z)$ from the channel, rather than just the samples from $R_{0}(z)$. This is the secret of its improved performance; the FSE "reads between lines," i.e., takes into account the samples of the effective channel between symbols. An obvious extension of the FSE scheme given above would be to use a finer spacing, e.g., $K$ times the symbol rate. Then, Fig. 23(a) and (b) would be modified as shown in Fig. 24, where $F_{K}(z)=G(z) C(z)_{\downarrow M / K}$ (assuming $K$ is a factor of $M$ ).

Many papers have been written on the topic of FSE. An excellent tutorial is the paper by Treichler et al. [17]. The work of Proakis, in [12, Sec. 10-2-4], is an insightful exposure and cites pioneering references. The papers by Tong et al. [15], [16] develop many theoretical results, some of which are applicable to the above context.

\section{CONCLUDING REMARKS}

Transfer function pairs called biorthogonal partners arise naturally in many signal processing applications. In this paper, we have provided a unified treatment of biorthogonal partners with some new results and taken a second look at some of the well-known results. Several applications were pointed out. This treatment has been possible because of contributions from many researchers in the filterbank and wavelet areas.

\section{REFERENCES}

[1] A. N. Akansu and R. A. Haddad, Multiresolution Signal Decomposition. New York: Academic, 1992.

[2] A. Aldroubi, "Oblique projections in atomic spaces," in Proc. Amer. Math. Soc., vol. 124, 1996, pp. 2051-2060.

[3] A. Aldroubi and M. Unser, "Oblique projections in discrete signal subspaces of $\ell_{2}$ and the wavelet transform," in Wavelet Applications in Signal and Image Processing, II. San Diego, CA: SPIE, vol. 2303, pp. $36-45$.

[4] A. Cohen and I. Daubechies, "A stability criterion for biorthogonal wavelet bases and their related subband coding scheme," Duke Math. J., vol. 68, no. 2, pp. 313-335, 1992.

[5] A. Cohen, I. Daubechies, and Feauveau, "Biorthogonal bases of compactly supported wavelets," Commun. Pure Appl. Math., vol. 45, no. 2, pp. 485-560, 1992.

[6] I. Daubechies, Ten Lectures on Wavelets. Philadelphia, PA: SIAM, Apr. 1992. 
[7] C. de Boor, A Practical Guide to Splines. New York: Springer-Verlag, 1978.

[8] I. Djokovic and P. P. Vaidyanathan, "Generalized sampling theorems in multiresolution subspaces," IEEE Trans. Signal Processing, vol. 45, pp. 583-599, Mar. 1997.

[9] S. Mallat, "A theory for multiresolution signal decomposition: the wavelet representation," IEEE Trans. Pattern Anal. Machine Intell., vol. 11, pp. 674-693, July 1989.

[10] — A Wavelet Tour of Signal Processing. New York: Academic, 1998.

[11] A. V. Oppenheim, A. S. Willsky, and I. T. Young, Signals and Systems. Englewood Cliffs, NJ: Prentice-Hall, 1983.

[12] J. G. Proakis, Digital Communications. New York: McGraw-Hill, 1995.

[13] I. J. Schoenberg, Cardinal Spline Interpolation. Philadelphia, PA: SIAM, 1973.

[14] M. G. Strintzis, "Optimal pyramidal and subband decompositions for hierarchical coding of noisy and quantized images," IEEE Trans. Image Processing, vol. 7, pp. 155-166, Feb. 1998.

[15] L. Tong, G. Xu, and T. Kailath, "Blind identification and equalization based on second order statistics: A time domain approach," IEEE Trans. Inform. Theory, vol. 40, pp. 340-349, Mar. 1994.

[16] — "Blind identification and equalization based on second order statistics: A frequency domain approach," IEEE Trans. Inform. Theory, vol. 41, pp. 329-334, Jan. 1995

[17] J. R. Treichler, I. Fijalkow, and C. R. Johnson, Jr., "Fractionally spaced equalizers: How long should they really be?," IEEE Signal Processing Mag., pp. 65-81, May 1996.

[18] M. Unser and A. Aldroubi, "Polynomial splines and wavelets," in Wavelets, A Tutorial in Theory and Applications, C. K. Chui, Ed. New York: Academic, 1992.

[19] M. Unser, A. Aldroubi, and M. Eden, "Fast B-spline transforms for continuous image representation and interpolation," IEEE Trans. Pattern Anal. Machine Intell., vol. 10, pp. 277-285, Mar. 1991.

[20] — , "B-spline signal processing-Part I: Theory," IEEE Trans. Signal Processing, vol. 41, pp. 821-833, Feb. 1993.

[21] — , "B-spline signal processing-Part II: Efficient design and applications," IEEE Trans. Signal Processing, vol. 41, pp. 834-848, Feb. 1993.

[22] M. Unser, "Sampling -50 years after Shannon," Proc. IEEE, vol. 88, pp. 569-587, Apr. 2000.

[23] P. P. Vaidyanathan, Multirate Systems and Filter Banks. Englewood Cliffs, NJ: Prentice-Hall, 1993

[24] P. P. Vaidyanathan and I. Djokovic, "Wavelet transforms," in The Cir cuits and Filters Handbook, W. K. Chen, Ed. Boca Raton, FL: CRC, 1995, pp. 134-219.

[25] P. P. Vaidyanathan and S.-M. Phoong, "Reconstruction of sequences from nonuniform samples," in Proc. IEEE Int. Symp. Circuits Syst., Seattle, WA, Apr--May 1995

[26] _ _ "Discrete time signals which can be recovered from samples," in Proc. IEEE Int. Conf. Acoust. Speech, Signal Process., Detroit, MI, May 1995.

[27] M. Vetterli and J. Kovačević, Wavelets and Subband Coding. Englewood Cliffs, NJ: Prentice-Hall, 1995.

[28] G. G. Walter, "A sampling theorem for wavelet subspaces," IEEE Trans. Inform. Theory, vol. 38, pp. 881-884, Mar. 1992.

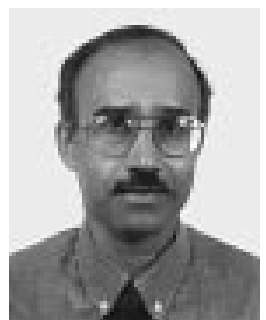

P. P. Vaidyanathan (S'80-M'83-SM'88-F'91) was born in Calcutta, India, on October 16, 1954. He received the B.Sc. (Hons.) degree in physics and the B.Tech. and M.Tech. degrees in radiophysics and electronics, all from the University of Calcutta, in 1974, 1977, and 1979, respectively, and the Ph.D. degree in electrical and computer engineering from the University of California, Santa Barbara (UCSB), in 1982

He was a Post Doctoral Fellow at UCSB from September 1982 to March 1983. In March 1983, he joined the Electrical Engineering Department, California Institute of Technology (Caltech), Pasadena, as an Assistant Professor, and since 1993, he has been Professor of Electrical Engineering. His main research interests are in digital signal processing, multirate systems, wavelet transforms, and signal processing for digital communications.

Dr. Vaidyanathan served as Vice Chairman of the Technical Program Committee for the 1983 IEEE International Symposium on Circuits and System and as the Technical Program Chairman for the 1992 IEEE International Symposium on Circuits and Systems. He was an Associate Editor for the IEEE TRANSACTIONS ON CIRCUITS AND SYSTEMS from 1985 to 1987 and is currently an Associate Editor for the IEEE SigNAl PROCESSING LETTERS and a Consulting Editor for the journal Applied and Computational Harmonic Analysis. He was a Guest Editor in 1998 for special issues of the IEEE TRANSACTIONS ON Signal Processing and the IEEE TRANSACTIONS ON CiRCUITS AND SYSTEMS II, on the topics of filterbanks, wavelets, and subband coders. He has authored a number of papers in IEEE journals and is the author of the book Multirate Systems and Filter Banks (Englewood Cliffs, NJ: Prentice-Hall, 1993). He has written several chapters for various signal processing handbooks. He was a recipient of the Award for Excellence in Teaching at the Caltech in 1983-1984, 1992-1993, and 1993-1994. He received the National Science Foundation Presidential Young Investigator award in 1986. In 1989, he received the IEEE ASSP Senior Award for his paper on multirate perfect-reconstruction filter banks. In 1990, he was recipient of the S. K. Mitra Memorial Award from the Institute of Electronics and Telecommunications Engineers, India, for his joint paper in the IETE journal. He was also the coauthor of a paper on linear-phase perfect reconstruction filter banks in the IEEE TRANSACTIONS ON SIGNAL PROCESSING, for which the first author (T. Nguyen) received the Young Outstanding $\mathrm{Au}$ thor award in 1993. He received the 1995 F. E. Terman Award of the American Society for Engineering Education, sponsored by Hewlett Packard Co., for his contributions to engineering education, especially the book Multirate Systems and Filter Banks. He has given several plenary talks at the Eusipco'98, Asimolar' 88 , and SPCOM'95 conferences on signal processing. He has been chosen a Distinguished Lecturer for the IEEE Signal Processing Society for the year 1996-1997. In 1999, he was chosen to receive the IEEE Circuits and Systems Society's Golden Jubilee Medal.

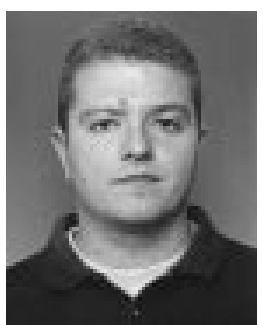

Bojan Vrcelj (S'99) was born in Belgrade, Yugoslavia, in 1974. He received the B.S. degree from the University of Belgrade in 1998 and the M.S. degree from the California Institute of Technology (Caltech), Pasadena, in 1999, both in electrical engineering. In 1998, he received the Graduate Division Fellowship from Caltech. He is currently pursuing the Ph.D. degree in the field of digital signal processing at Caltech.

His research interests include multirate systems, wavelets and their applications in digital communications, as well as image processing and interpolation. 